\title{
Highly Diastereoselective Epoxidation of Allylically Substituted Cycloalkenes Catalyzed by Metalloporphyrins
}

\author{
Wing-Kei Chan, Peng Liu, Wing-Yiu Yu, Man-Kin Wong, * and Chi-Ming Che* \\ Department of Chemistry and Open Laboratory of Chemical Biology of the Ins titute of \\ Molecular Technology for Drug Discovery and Synthesis, The University of Hong Kong, \\ Pokfulam Road, Hong Kong, China \\ cmche@hku.hk
}

\section{SUPPORTING INFORMATION}

\section{Table of Contents}

General Structures of Metalloporphyrin Catalysts

$\mathrm{S} 2$

Control Experiments for Diastereoselective Epoxidationof $\mathbf{3 d}, \mathbf{3 f}$ and $\mathbf{3 h}$

S3- S4

Literature References for Cycloalkenes $\mathbf{3 b}-\mathbf{3 d}, \mathbf{3 f}-\mathbf{3 g}, \mathbf{3 i}$ and $\mathbf{3 l}-\mathbf{3} \mathbf{p}$

S5-S6

Preparation of Cycloalkenes $\mathbf{3 h}, \mathbf{3} \mathbf{j}$ and $\mathbf{3 k}$

S7-S8

Literature References for Epoxides 4a-4g, 4i and 4m-4p

S9-S10

Characterization Data of Epoxides $4 \mathbf{h}$ and $\mathbf{4 j - 4 l}$

S11-S13

GC Spectra of Diastereomeric Mixture of $4 \mathbf{c}$

S14

${ }^{1} \mathrm{H}$ NMR Spectra of Diastereomeric Mixtures of Epoxides 4

S15-S23

${ }^{1} \mathrm{H}$ NMR Spectrum of $\mathbf{3 h}, \mathbf{3} \mathbf{j}$ and $\mathbf{3 k}$

S24-S26

${ }^{13} \mathrm{C}$ NMR Spectrum of $\mathbf{3 h}, \mathbf{3} \mathbf{j}$ and $\mathbf{3 k}$

S27-S39

${ }^{1} \mathrm{H}$ NMR Spectrum of $\mathbf{4 h}$ and $\mathbf{4 j}-\mathbf{4 l}$

S30-S35

${ }^{13} \mathrm{C}$ NMR Spectrum of $\mathbf{4 h}$ and $\mathbf{4 j - 4 l}$

S36-S41 


\section{General Structures of Metalloporphyrin Catalysts}

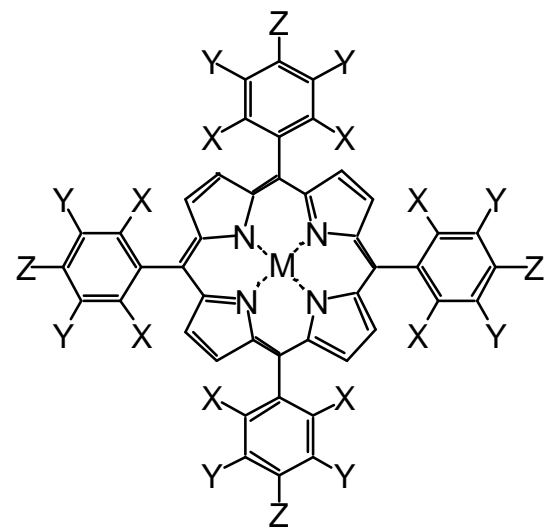

$$
\begin{array}{rlrl}
\text { 1: } \mathrm{M}=\mathrm{Mn}^{3+} ; \mathrm{X}=\mathrm{Cl} ; \mathrm{Y}=\mathrm{Z}=\mathrm{H} & \mathrm{Mn}(\mathrm{TDCPP}) \mathrm{Cl} \\
\text { 2: } \mathrm{M}=\mathrm{Ru}^{2+} ; \mathrm{X}=\mathrm{Cl} ; \mathrm{Y}=\mathrm{Z}=\mathrm{H} & \mathrm{Ru}(\mathrm{TDCPP})(\mathrm{CO}) \\
\mathrm{M}=\mathrm{Mn}^{3+} ; \mathrm{X}=\mathrm{CH}_{3} ; \mathrm{Y}=\mathrm{Z}=\mathrm{H} & \mathrm{Mn}(\mathrm{TMP}) \mathrm{Cl} \\
\mathrm{M}=\mathrm{Mn}^{3+} ; \mathrm{X}=\mathrm{Y}=\mathrm{Z}=\mathrm{F} & \mathrm{Mn}(\mathrm{TPFPP}) \mathrm{Cl} \\
\mathrm{M}=\mathrm{Mn}^{3+} ; \mathrm{X}=\mathrm{Y}=\mathrm{H} ; \mathrm{Z}=\mathrm{CH}_{3} & \mathrm{Mn}(\mathrm{TTP}) \mathrm{Cl}
\end{array}
$$




\section{Control Experiments for Diastereoselective Epoxidation of 3d, 3f and $3 \mathrm{~h}$}

In table 1, it is noticed that high trans-selectivities yet moderate yields were obtained in the 1-catalyzed epoxidation of $\mathbf{3 d}, \mathbf{3 f}$, and $\mathbf{3 h}$. To exclude the possibility that the high transselectivities might come from preferential decomposition of one of the diastereomeric epoxides during the reaction conditions, several control experiments have been performed. Epoxides $\mathbf{4 d}$, 4f, and $\mathbf{4 h}$ with known trans-selectivities (i.e., trans- : cis-epoxide ratio) were individually subjected to the 1-catalyzed epoxidation reaction conditions. After the reactions, transselectivities of the recovered epoxides were determined by ${ }^{1} \mathrm{H}$ NMR. The results are summarized as follows:

\begin{tabular}{|c|c|c|c|}
\hline trans-4 & cis-4 & $\begin{array}{l}\text { DCPP)Cl] (1) } \\
\text { aq } \mathrm{NH}_{4} \mathrm{HCO}_{3} \\
\mathrm{~N} \text {, r.t., } 2.5 \mathrm{~h}\end{array}$ & $\overbrace{\text { trans }-4}^{R_{1}}+\underbrace{R_{1}}_{c i s-4}$ \\
\hline entry & $\begin{array}{c}\text { Epoxide } \\
\text { (trans:cis ratio) }\end{array}$ & $\begin{array}{c}\% \text { of } \\
\text { recovery }^{b}\end{array}$ & $\begin{array}{c}\text { Trans/cis ratio of } \\
\text { recovered epoxide }\end{array}$ \\
\hline 1 & $\mathbf{4 d}(4.6: 1)$ & 84 & $4.8: 1$ \\
\hline 2 & $\mathbf{4 f}(1.4: 1)$ & 92 & $1.4: 1$ \\
\hline 3 & $4 h(5: 1)$ & 74 & 4:1 \\
\hline \multicolumn{4}{|c|}{$\begin{array}{l}\text { all the epoxidation reactions were performed as follows: A } \\
\text { solution of epoxide }(0.25 \mathrm{mmol}) \text { and } 1(3 \mu \mathrm{mol}) \text { in } \mathrm{CH}_{3} \mathrm{CN}(4 \\
\mathrm{mL}) \text { was added a pre-mixed solution of } 0.8 \mathrm{M} \text { aqueous } \mathrm{NH}_{4} \mathrm{HCO}_{3} \\
(0.5 \mathrm{~mL}), \mathrm{CH}_{3} \mathrm{CN}(0.5 \mathrm{~mL}) \text { and } 35 \% \mathrm{H}_{2} \mathrm{O}_{2}(0.10 \mathrm{~mL}) \text { at room } \\
\text { temperature. }{ }^{b} \text { Determined by }{ }^{1} \mathrm{H} \mathrm{NMR} \text { with internal standard. }\end{array}$} \\
\hline
\end{tabular}

As illustrated in the above table, no significant change on the trans-selectivities of the recovered epoxides 4d, $4 \mathbf{f}$ and $\mathbf{4 h}$ was observed. These findings indicated that the high trans-selectivities observed in epoxidation of $\mathbf{3 d}, \mathbf{3 f}$ and $\mathbf{3 h}$ should not come from preferential decomposition of one of the diastereomeric epoxides. 


\section{Procedure for Control Experiment for Diastereoselective Epoxidation of 3d}

To a round-bottom flask containing $\mathrm{Mn}(\mathrm{TDCPP})(\mathrm{Cl}) \mathbf{1}(3.0 \mathrm{mg}, 0.003 \mathrm{mmol})$ and $\mathbf{4 d}$ (trans cis ratio $=4.6: 1)(88.0 \mathrm{mg}, 0.25 \mathrm{mmol})$ in $\mathrm{CH}_{3} \mathrm{CN}(4 \mathrm{~mL})$ was added a premixed solution of $30 \% \mathrm{H}_{2} \mathrm{O}_{2}(0.10 \mathrm{~mL})$, aqueous $\mathrm{NH}_{4} \mathrm{HCO}_{3}(0.8 \mathrm{M}, 0.5 \mathrm{~mL})$ and $\mathrm{CH}_{3} \mathrm{CN}(0.5 \mathrm{~mL})$ via a syringe pump for $1.5 \mathrm{~h}$ at room temperature. After being stirred for $1 \mathrm{~h}$, the reaction mixture was diluted with saturated aqueous $\mathrm{Na}_{2} \mathrm{~S}_{2} \mathrm{O}_{3}(1 \mathrm{~mL})$ and extracted with $\mathrm{n}$-hexane $(4 \times 20 \mathrm{~mL})$. The combined organic layers were dried over anhydrous $\mathrm{MgSO}_{4}$, filtered through a short pad of silica gel, and concentrated under reduced pressure. The $\%$ of recovery and ratio of trans-4d to cis-4d was determined to be $84 \%$ and 4.8:1 respectively, by ${ }^{1} \mathrm{H}$ NMR with internal standard. 


\section{Literature References for Cycloalkenes 3b-3d, 3f-3g, 3i, and 31-3p}

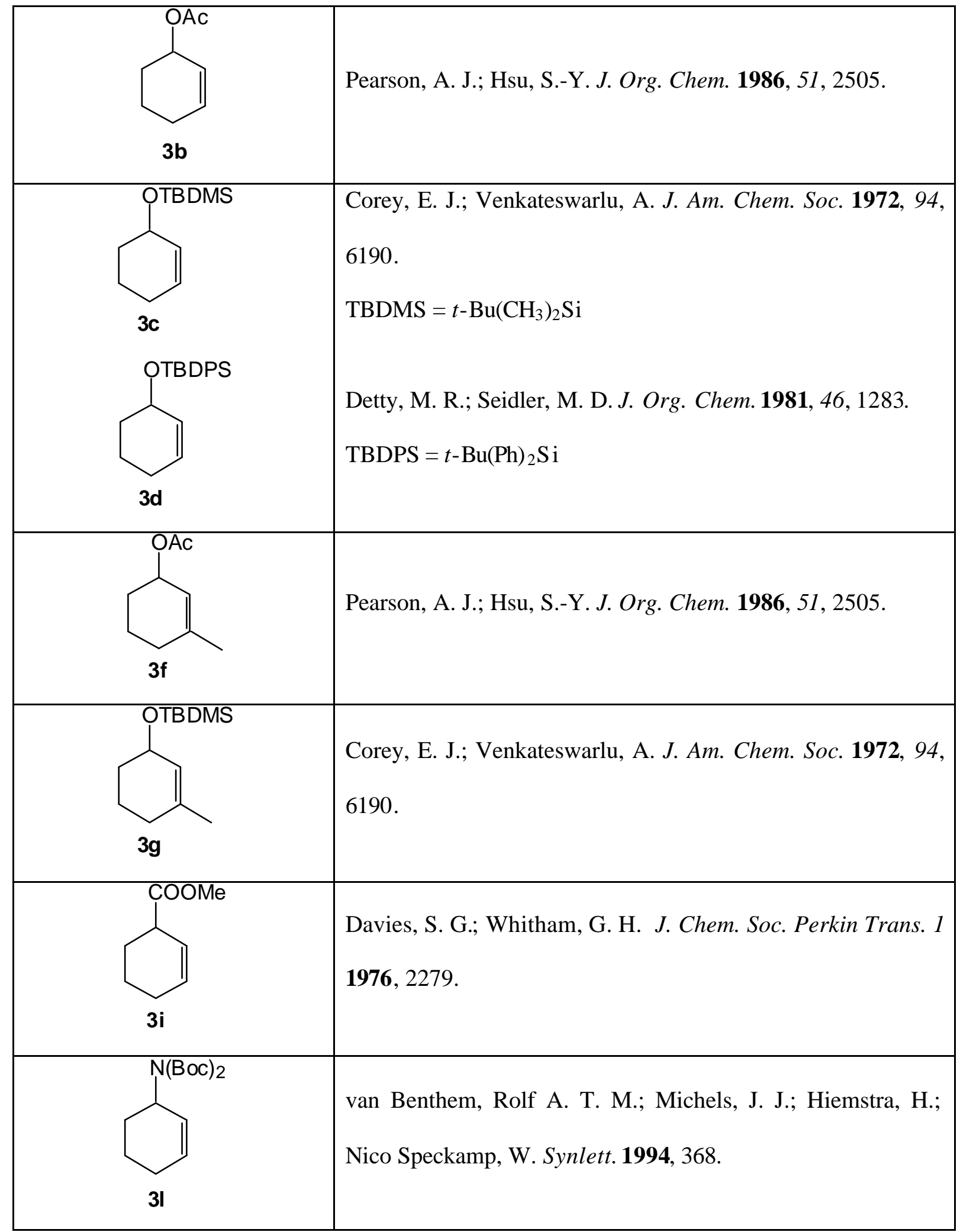




\begin{tabular}{|l|l|}
\hline Crotti, P.; Di Bussolo, V.; Favero, L.; Macchia, F.; Pineschi, \\
\hline M. Eur. J. Org. Chem. 1998, 1675.
\end{tabular}




\section{Preparation of Cycloalkenes 3h, 3j and 3k}

\section{Preparation of $\mathbf{3 h}$}

A solution of 3-methyl-2-cyclohexen-1-ol (0.49 g, 5 mmol), TBDPSCl (1.01 g, $5.5 \mathrm{mmol})$, imidazole $(0.5 \mathrm{~g}, 7.3 \mathrm{mmol})$ in anhydrous DMF (5 mL) was stirred at room temperature for $16 \mathrm{~h}$ The mixture was diluted with EtOAc $(50 \mathrm{~mL})$, washed with $1 \mathrm{~N} \mathrm{HCl}$, saturated $\mathrm{NaHCO}_{3}$ solution and brine, and concentrated under reduced pressure. The residue was purified by flash column chromatography (1\% EtOAc in hexane) to afford alkene $\mathbf{3 h}(1.4 \mathrm{~g}, 4.0 \mathrm{mmol}, 80 \%$ yield). Colorless oil, analytical TLC (silica gel 60) (10\% EA in hexane), $\mathrm{R}_{f}=0.58 ;{ }^{1} \mathrm{H}$ NMR $(400 \mathrm{MHz}$, $\left.\mathrm{CDCl}_{3}\right) \mathrm{d}$ 7.70-7.68 (m, 4H), 7.43-7.24 (m, 6H), $5.35(\mathrm{~m}, 1 \mathrm{H}), 4.21(\mathrm{~m}, 1 \mathrm{H}), 1.94-1.79(\mathrm{~m}, 1 \mathrm{H})$, $1.78-1.72(\mathrm{~m}, 2 \mathrm{H}), 1.61(\mathrm{~s}, 3 \mathrm{H}), 1.60-1.53(\mathrm{~m}, 2 \mathrm{H}), 1.46-1.37(\mathrm{~m}, 1 \mathrm{H}), 1.06(\mathrm{~s}, 9 \mathrm{H}) ;{ }^{13} \mathrm{C} \mathrm{NMR}$ (75.47 MHz, $\left.\mathrm{CDCl}_{3}\right) \mathrm{d} 136.91,135.87,135.82,134.79,134.73,129.41,129.39,127.44,127.42$, 125.32, 67.83, 31.93, 30.01, 27.05, 23.59, 19.58, 19.20; IR (KBr) 2931, 1472, $821 \mathrm{~cm}^{-1}$; EIMS $m / z 350\left(\mathrm{M}^{+}\right), 293\left(\mathrm{M}^{+}-t \mathrm{C}_{4} \mathrm{H}_{9}\right)$; HRMS (EI) for $\mathrm{C}_{23} \mathrm{H}_{30} \mathrm{OSi}$, calcd 350.2066, found 350.2062.

\section{Preparation of $\mathbf{3 j}$}

A solution of cyclohex-2-enecarboxylic acid (0.4 g, $3.2 \mathrm{mmol})$, cyclohexanol (0.635 g, 6.4 mmol), DMAP (0.195 g, $1.6 \mathrm{mmol})$, EDCI $0.92 \mathrm{~g}, 4.8 \mathrm{mmol})$ in anhydrous $\mathrm{CH}_{2} \mathrm{Ch}_{2}(10 \mathrm{~mL})$ were stirred at room temperature for $6 \mathrm{~h}$. The reaction mixture was diluted with $\mathrm{CH}_{2} \mathrm{Cl}_{2}(70 \mathrm{~mL})$, washed with $\mathrm{HO}(2 \times 10 \mathrm{~mL})$, and dried over anhydrous $\mathrm{Na}_{2} \mathrm{SO}_{4}$. The reaction mixture was filtered, and concentrated under reduced pressure. The residue was purified by flash column chromatography (3\% EtOAc in hexane) to afford alkene $3 \mathbf{j}(0.5 \mathrm{~g}, 2.4 \mathrm{mmol}, 75 \%$ yield). Colorless oil, analytical TLC (silica gel 60) (10\% EA in hexane), $\mathrm{R}_{f}=0.50 ;{ }^{1} \mathrm{H}$ NMR $(300 \mathrm{MHz}$ 
$\left.\mathrm{CDCl}_{3}\right)$ d $5.85-5.74(\mathrm{~m}, 2 \mathrm{H}), 4.81-4.77(\mathrm{~m}, 1 \mathrm{H}), 3.06(\mathrm{~m}, 1 \mathrm{H}), 2.02(\mathrm{~m}, 2 \mathrm{H}), 1.92-1.64(\mathrm{~m}, 7 \mathrm{H})$, $1.60-1.26(\mathrm{~m}, 7 \mathrm{H}) ;{ }^{13} \mathrm{C}$ NMR $\left(75.47 \mathrm{MHz}, \mathrm{CDCl}_{3}\right) \mathrm{d} 174.00,129.36,124.65,72.36,41.39,31.51$, 25.43, 25.32, 24.67, 23.59, 20.80; IR (KBr) $1722 \mathrm{~cm}^{-1}$; EIMS m/z $208\left(\mathrm{M}^{+}\right)$; HRMS (EI) for $\mathrm{C}_{13} \mathrm{H}_{20} \mathrm{O}_{2}$, calcd 208.1463, found 208.1443. (Synthesis of cyclohex-2-enecarboxylic acid, see: Davies, S. G.; Whitham, G. H. J. Chem. Soc. Perkin Trans. 1 1976, 2279.)

\section{Preparation of $\mathbf{3 k}$}

A solution of cyclohex-2-enecarboxylic acid (0.48 g, $3.8 \mathrm{mmol})$, Benzhydrol (1.4 g, 7.6 mmol), DMAP (0.23 g, $1.9 \mathrm{mmol})$, EDCI $(1.1 \mathrm{mg}, 5.7 \mathrm{mmol})$ and anhydrous $\mathrm{CH}_{2} \mathrm{Cl}_{2}(10 \mathrm{~mL})$ were stirred at room temperature for 6 h. The reaction mixture was diluted with $\mathrm{CH}_{2} \mathrm{Cl}_{2}(70 \mathrm{~mL})$, washed with $\mathrm{H}_{2} \mathrm{O}(2 \times 10 \mathrm{~mL})$, and over anhydrous $\mathrm{Na}_{2} \mathrm{SO}_{4}$. The reaction mixture was filtered, and concentrated under reduced pressure. The residue was purified by flash column chromatography (3\% EtOAc in hexane) to afford alkene 3k (0.77 g, $2.6 \mathrm{mmol}, 69 \%$ yield). Colorless oil, analytical TLC (silica gel 60) (10\% EA in hexane), $\mathrm{R}_{f}=0.58 ;{ }^{1} \mathrm{H}$ NMR $(300 \mathrm{MHz}$, $\left.\mathrm{CDCl}_{3}\right)$ d $7.34-7.26(\mathrm{~m}, 10 \mathrm{H}), 6.88(\mathrm{~s}, 1 \mathrm{H}), 5.86-5.83(\mathrm{~m}, 2 \mathrm{H}), 3.22-3.20(\mathrm{~m}, 1 \mathrm{H}), 2.04-1.75(\mathrm{~m}$, 5H), $1.63-1.56(\mathrm{~m}, 1 \mathrm{H}) ;{ }^{13} \mathrm{C}$ NMR $\left(75.47 \mathrm{MHz}, \mathrm{CDCl}_{3}\right) \mathrm{d} 173.77,140.85,140.80,130.25$, $128.91,128.26,128.24,127.49,127.42,124.50,77.20,41.72,25.67,25.07,21.18 ; \mathrm{IR}(\mathrm{KBr})$ $1715 \mathrm{~cm}^{-1}$; EIMS m/z $292\left(\mathrm{M}^{+}\right), 167\left(\mathrm{M}^{+}-\mathrm{C}_{7} \mathrm{H}_{9} \mathrm{O}_{2}\right)$; HRMS (EI) for $\mathrm{C}_{20} \mathrm{H}_{20} \mathrm{O}_{2}$, calcd 292.1463, found 292.1455 . 


\section{Literature References for Epoxides $4 a-4 g$, $4 i$ and $4 m-4 p$}

\begin{tabular}{|l|l|}
\hline M. J. Org. Chem. 1996, 61, 1830. \\
\hline M. J. Org. Chem. 1996, 61, 1830.
\end{tabular}




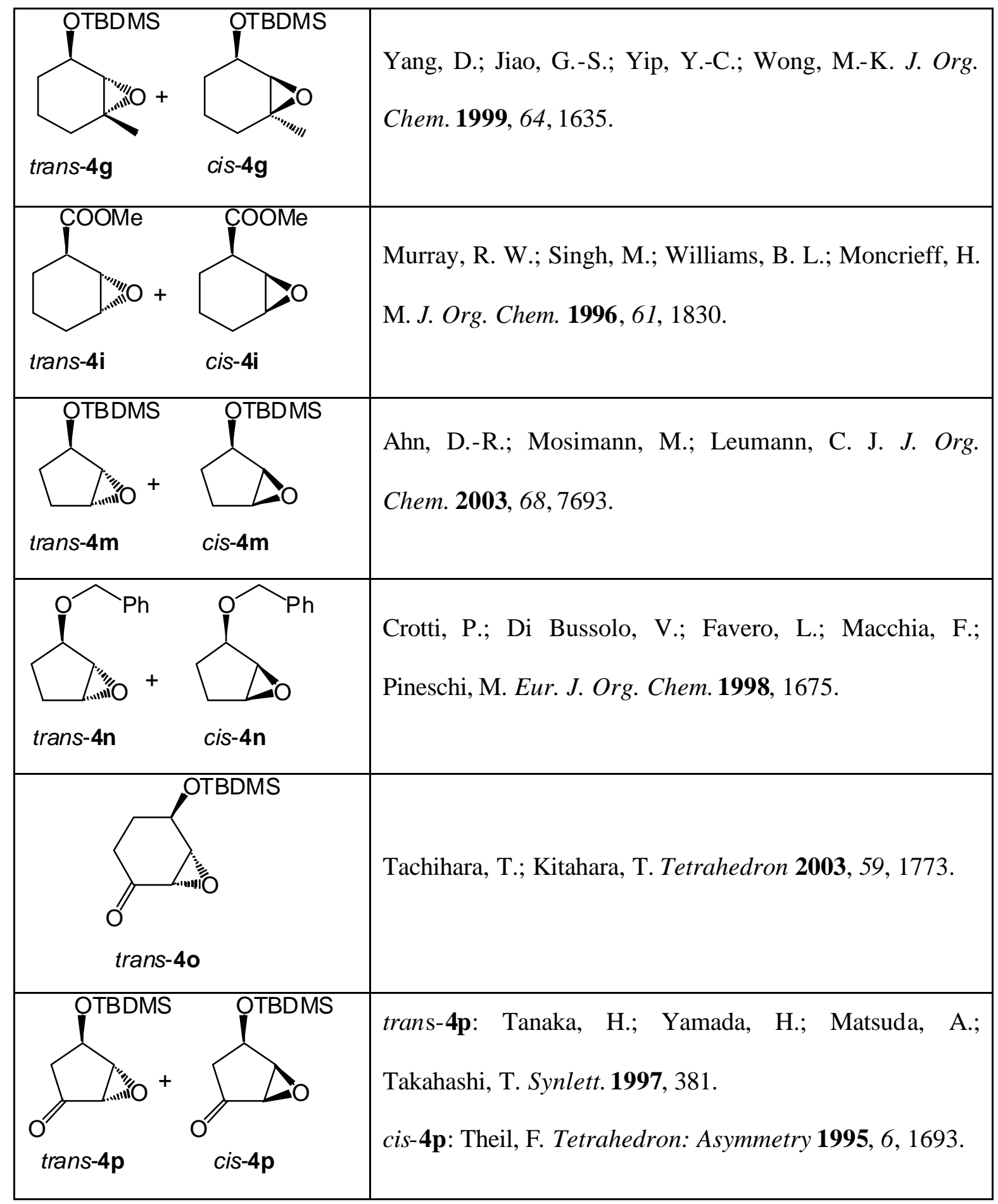




\section{Characterization Data of epoxide $4 \mathrm{~h}$, and $4 \mathrm{j}-4 \mathrm{l}$}

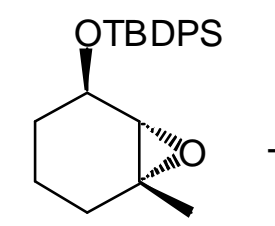

trans $-\mathbf{4 h}$

4

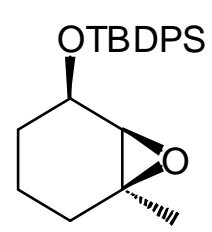

cis-4h

Colorless oil, analytical TLC (silica gel 60) (10\% EA in hexane), trans-4h $\mathrm{R}_{f}=0.28$, cis-4h $\mathrm{R}_{f}=$ 0.25; ${ }^{1} \mathrm{H}$ NMR (400 MHz, $\left.\mathrm{CDCl}_{3}\right)$ d 7.96-7.66 (m, 4H), 7.45-7.34 (m, 6H), 4.02-3.96 (m, 1H), $2.93($ br s, $4 / 5 \times 1 \mathrm{H}), 2.87($ br s, $1 / 5 \times 1 \mathrm{H}), 1.85-1.35(\mathrm{~m}, 5 \mathrm{H}), 1.29(\mathrm{~s}, 4 / 5 \times 3 \mathrm{H}), 1.26-1.13(\mathrm{~m}$, $5 \mathrm{H}), 1.22(\mathrm{~s}, 1 / 5 \times 3 \mathrm{H}), 1.09(\mathrm{~s}, 4 / 5 \times 9 \mathrm{H}), 1.08(\mathrm{~s}, 1 / 5 \times 9 \mathrm{H}) ;{ }^{13} \mathrm{C} \mathrm{NMR}\left(100.61 \mathrm{MHz}, \mathrm{CDCl}_{3}\right) \mathrm{d}$ $135.78,135.70,134.04,133.86,129.68,129.65,129.56,127.62,127.55,127.52,29.96,29.39$, 28.04, 27.66, 26.95, 26.89, 24.05, 23.36, 19.70, 19.17, 15.68; IR (KBr) 2933, 1472, $822 \mathrm{~cm}^{-1}$; EIMS $m / z 366\left(\mathrm{M}^{+}\right), 309\left(\mathrm{M}^{+}-t \mathrm{C}_{4} \mathrm{H}_{9}\right)$; HRMS (EI) for $\mathrm{C}_{23} \mathrm{H}_{30} \mathrm{O}_{2} \mathrm{Si}$, calcd 366.2015, found 366.2015 .

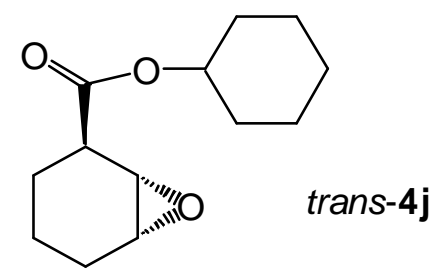

Colorless oil, analytical TLC (silica gel 60) (10\% EA in hexane), $\mathrm{R}_{f}=0.31 ;{ }^{1} \mathrm{H}$ NMR $(400 \mathrm{MHz}$, $\left.\mathrm{CDCl}_{3}\right) \mathrm{d} 4.86-4.80(\mathrm{~m}, 1 \mathrm{H}), 3.41(\mathrm{~d}, J=3.9 \mathrm{~Hz}, 1 \mathrm{H}), 3.22-3.20(\mathrm{~m}, 1 \mathrm{H}), 2.87-2.84(\mathrm{dd}, J=8.6$, 5.6 Hz, 1H), 2.08-2.03 (m, 1H), 1.85-1.69 (m, 6H), 1.57-1.25 (m, 9H); ${ }^{13} \mathrm{C}$ NMR (100.62 MHz, $\left.\mathrm{CDCl}_{3}\right) \mathrm{d} 172.97,72.80,52.36,52.24,40.91,31.49,31.43,25.32,23.94,23.78,23.55,23.53$, 
16.80; IR (KBr) $1728 \mathrm{~cm}^{-1}$; EIMS m/z, $224\left(\mathrm{M}^{+}\right)$; HRMS (EI) for $\mathrm{C}_{3} \mathrm{H}_{20} \mathrm{O}_{3}$, calcd 224.1412, found 224.14037 .

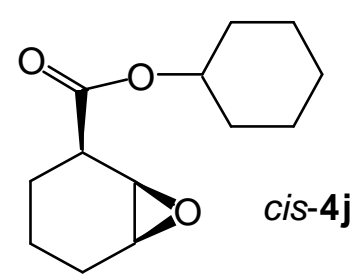

Colorless oil, analytical TLC (silica gel 60) (10\% EA in hexane), $\mathrm{R}_{f}=0.25 ;{ }^{1} \mathrm{H}$ NMR $(400 \mathrm{MHz}$ $\left.\mathrm{CDCl}_{3}\right)$ d $4.88-4.84(\mathrm{~m}, 1 \mathrm{H}), 3.45(\mathrm{t}, J=3.5 \mathrm{~Hz}, 1 \mathrm{H}), 3.21-3.19(\mathrm{~m}, 1 \mathrm{H}), 2.83-2.78(\mathrm{~m}, 1 \mathrm{H})$, 1.90-1.82 (m, 4H), 1.76-1.67 (m, 3H), 1.61-1.23 (m, 9H); ${ }^{13} \mathrm{C}$ NMR (100.62 MHz, CDCl $) \mathrm{d}$ $172.24,72.75,52.26,52.18,41.13,31.50,31.47,25.40,23.53,23.58,23.35,21.29,18.91$; IR (KBr) $1734 \mathrm{~cm}^{-1}$; EIMS m/z $125\left(\mathrm{M}^{+}-\mathrm{C}_{6} \mathrm{H}_{11} \mathrm{O}\right.$ ); HRMS (EI) for $\mathrm{C}_{7} \mathrm{H}_{9} \mathrm{O}_{2}$, calcd 125.0603, found 125.0602 .

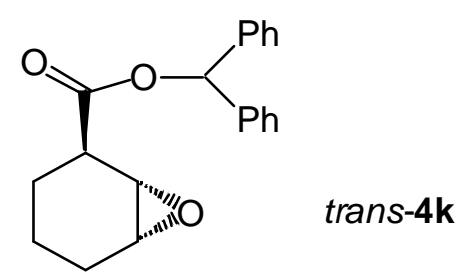

Colorless oil, analytical TLC (silica gel 60) (20\% EA in hexane), $\mathrm{R}_{f}=0.38 ;{ }^{1} \mathrm{H}$ NMR $(300 \mathrm{MHz}$, $\left.\mathrm{CDCl}_{3}\right) \mathrm{d} 7.35-7.26(\mathrm{~m}, 10 \mathrm{H}), 6.92(\mathrm{~s}, 1 \mathrm{H}), 3.45(\mathrm{~d}, 3.5 \mathrm{~Hz}, 1 \mathrm{H}), 3.21-3.19(\mathrm{~m}, 1 \mathrm{H}), 3.01(\mathrm{dd}, J$ $=8.8,6.5 \mathrm{~Hz}, 1 \mathrm{H}), 2.08-2.01(\mathrm{~m}, 1 \mathrm{H}), 1.91-1.87(\mathrm{~m}, 1 \mathrm{H}), 1.86-1.70(\mathrm{~m}, 1 \mathrm{H}), 1.49-1.33(\mathrm{~m}, 3 \mathrm{H})$; ${ }^{13} \mathrm{C}$ NMR $\left(75.47 \mathrm{MHz}, \mathrm{CDCl}_{3}\right) \mathrm{d} 172.85,140.44,140.37,128.97,128.95,128.43,128.38,127.46$, 127.36, 77.60, 52.65, 52.56, 41.26, 24.29, 24.12, 17.17; IR (KBr) $1732 \mathrm{~cm}^{-1}$; EIMS m/z $308\left(\mathrm{M}^{+}\right)$, $183\left(\mathrm{M}^{+}-\mathrm{C}_{7} \mathrm{H}_{9} \mathrm{O}_{2}\right)$; HRMS (EI) for $\mathrm{C}_{20} \mathrm{H}_{20} \mathrm{O}_{3}$, calcd 308.1412, found 308.1407. 


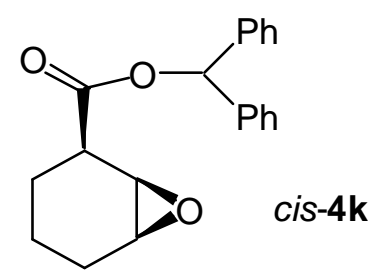

Colorless oil, analytical TLC (silica gel 60) (20\% EA in hexane), $\mathrm{R}_{f}=0.30 ;{ }^{1} \mathrm{H}$ NMR $(300 \mathrm{MHz}$, $\left.\mathrm{CDCl}_{3}\right)$ d 7.39-7.23 (m, 10H), $6.94(\mathrm{~s}, 1 \mathrm{H}), 3.54(\mathrm{t}, J=3.4 \mathrm{~Hz}, 1 \mathrm{H}), 3.24-3.21(\mathrm{~m}, 1 \mathrm{H}), 2.95-$ $2.89(\mathrm{~m}, 1 \mathrm{H}), 1.91-1.85(\mathrm{~m}, 2 \mathrm{H}), 1.80-1.64(\mathrm{~m}, 1 \mathrm{H}), 1.61-1.57(\mathrm{~m}, 2 \mathrm{H}), 1.29-1.22(\mathrm{~m}, 1 \mathrm{H}) ;{ }^{13} \mathrm{C}$ NMR (75.47 MHz, $\left.\mathrm{CDCl}_{3}\right)$ d 171.79, 140.20, 140.13, 128.44, 128.40, 127.87, 127.71, 127.20, 126.82, 77.14, 52.12, 51.95, 41.27, 23.23, 21.32, 18.90; IR (KBr) $1738 \mathrm{~cm}^{-1}$; EIMS m/z $308\left(\mathrm{M}^{+}\right)$, $183\left(\mathrm{M}^{+}-\mathrm{C}_{7} \mathrm{H}_{9} \mathrm{O}_{2}\right)$; HRMS (EI) for $\mathrm{C}_{20} \mathrm{H}_{20} \mathrm{O}_{3}$, calcd 308.1412, found 308.1407.

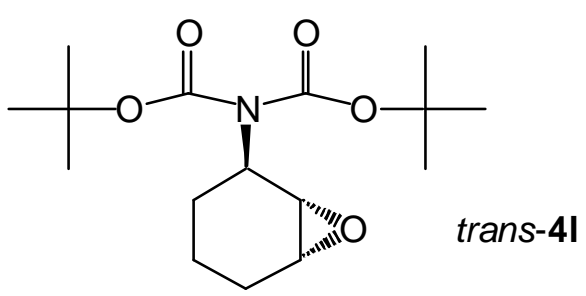

Colorless oil; analytical TLC (silica gel 60) (30\% EA in hexane), $\mathrm{R}_{f}=0.67 ;{ }^{1} \mathrm{H} \mathrm{NMR}(300 \mathrm{MHz}$, $\left.\mathrm{CDCl}_{3}\right) \mathrm{d} 4.32(\mathrm{dd}, J=10.8,6.3 \mathrm{~Hz}, 1 \mathrm{H}), 3.29(\mathrm{~m}, 1 \mathrm{H}), 3.22(\mathrm{~d}, J=3.9 \mathrm{~Hz}, 1 \mathrm{H}), 2.12-2.07(\mathrm{~m}$, 1H), 1.79-1.68 (m, 2H), $1.52(\mathrm{~s}, 18 \mathrm{H}), 1.48-1.43(\mathrm{~m}, 2 \mathrm{H}), 1.42-1.26(\mathrm{~m}, 1 \mathrm{H}) ;{ }^{13} \mathrm{C}$ NMR $(75.47$ $\mathrm{MHz}, \mathrm{CDCl}_{3}$ ) d 152.97, 83.31, 57.54, 54.18, 53.25, 28.57, 25.90, 24.68, 16.81; IR (KBr) 1738, $1698 \mathrm{~cm}^{-1}$; EIMS $m / z 257\left(\mathrm{M}^{+}+1-t \mathrm{C}_{4} \mathrm{H}_{9}\right)$; HRMS (EI) for $\mathrm{C}_{12} \mathrm{H}_{19} \mathrm{O}_{5} \mathrm{~N}\left(\mathrm{M}^{+}+1-\mathrm{C}_{4} \mathrm{H}_{9}\right)$, calcd 257.1263, found 257.1261. 
GC spectra of Diastereomeric mixture of $4 c$

Table 1, entry 3
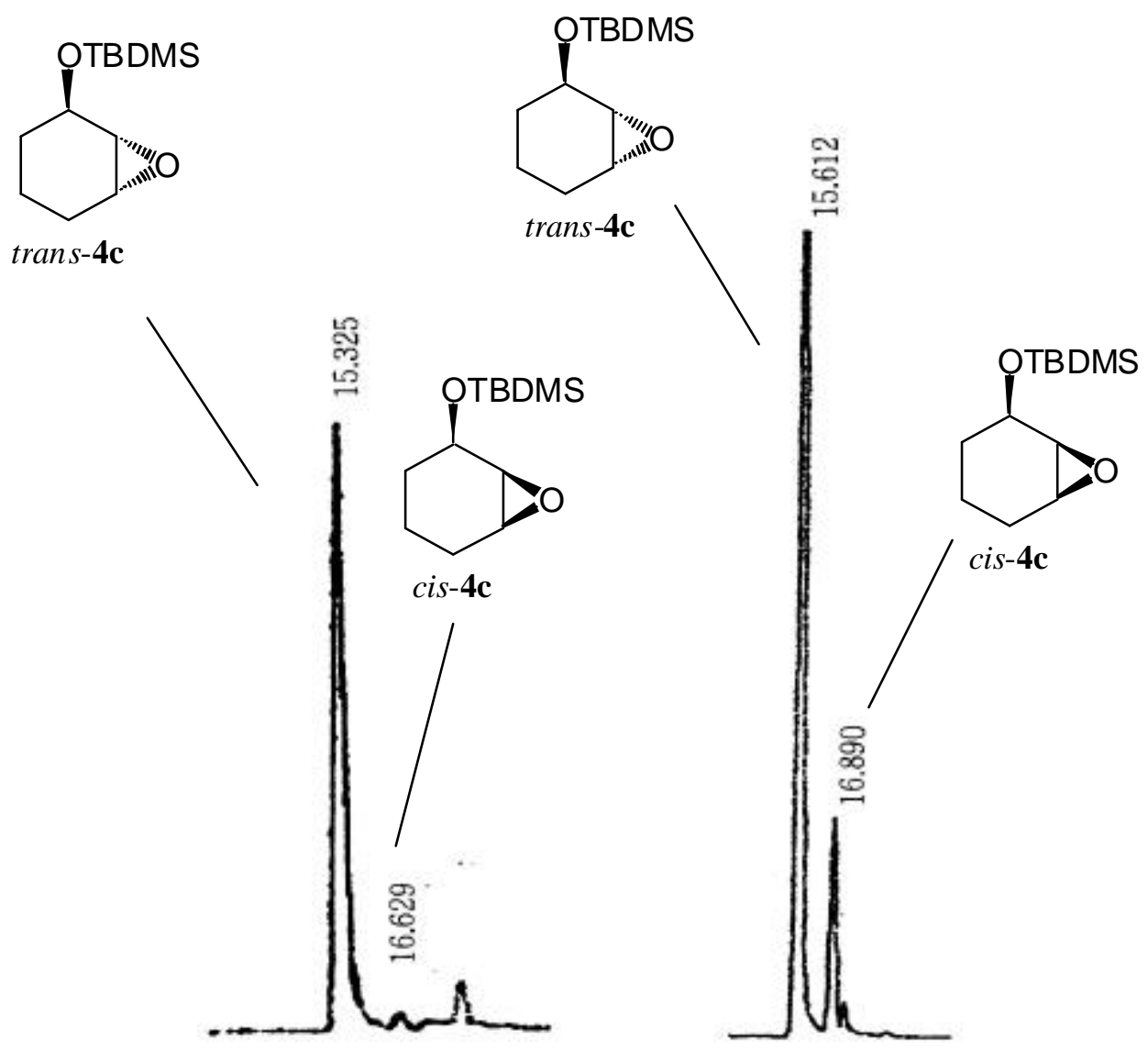

trans $:$ cis $=33: 1$

trans $: \operatorname{cis}=5: 1$ 


\section{${ }^{1} \mathrm{H}$ NMR Spectra of Diastereomeric Mixtures of Epoxide 4}

Table 1, entry 4

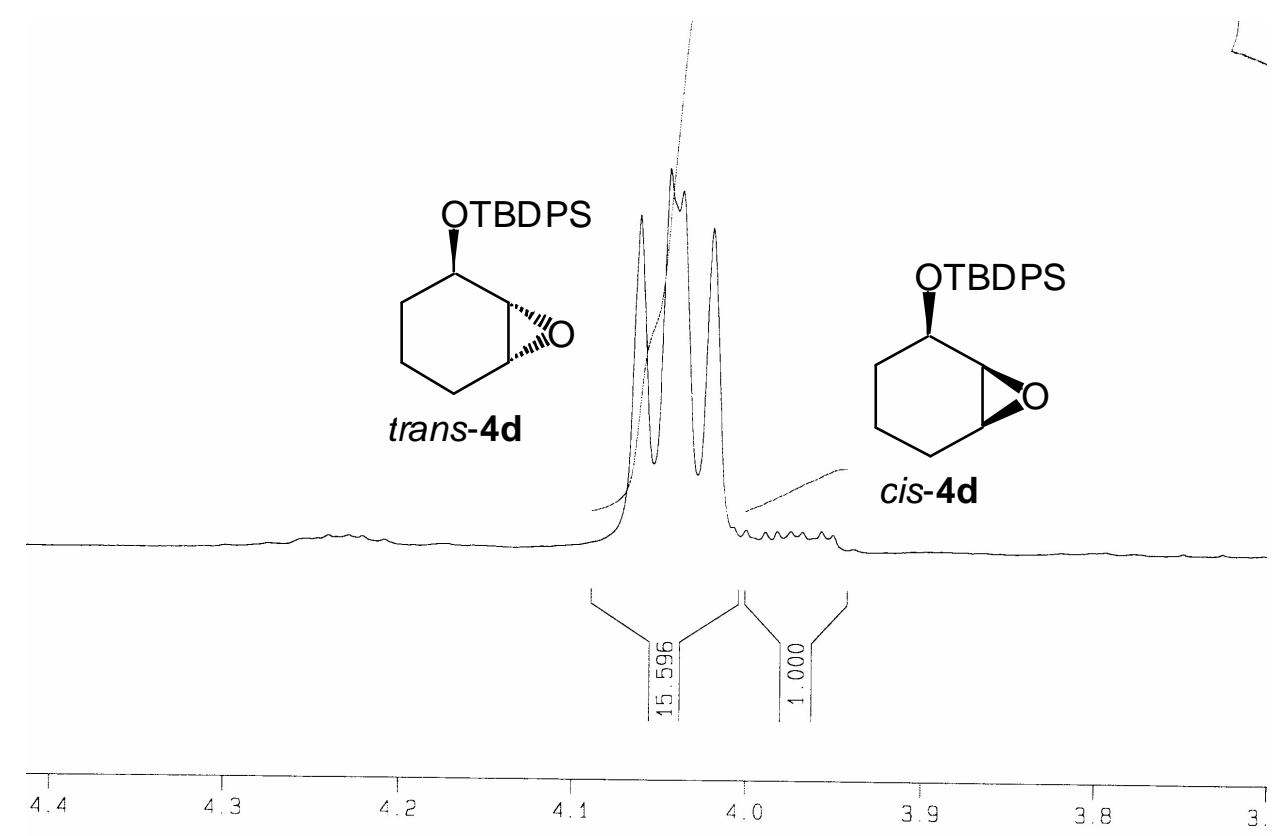

Table 1, entry 6

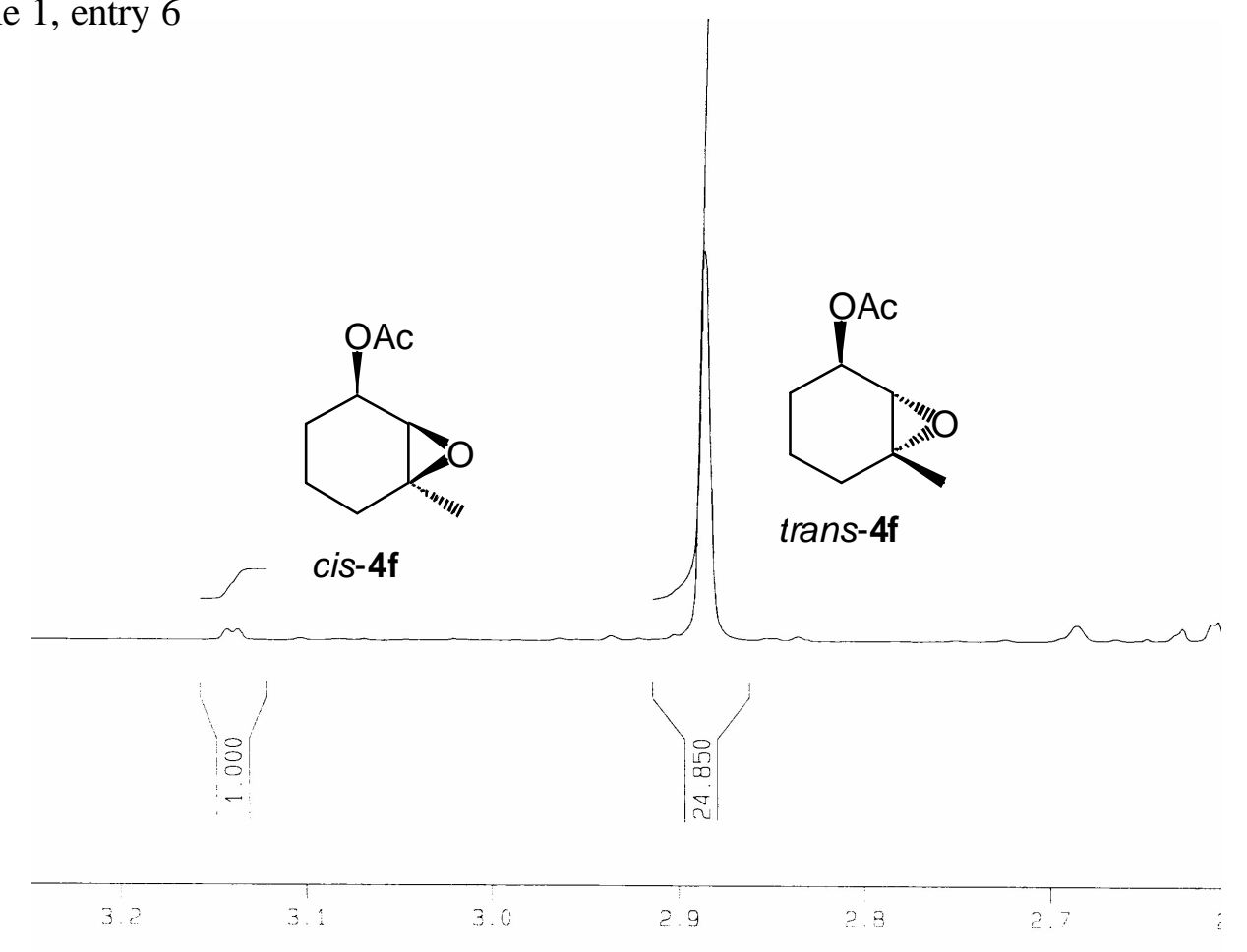


Table 1, entry 7
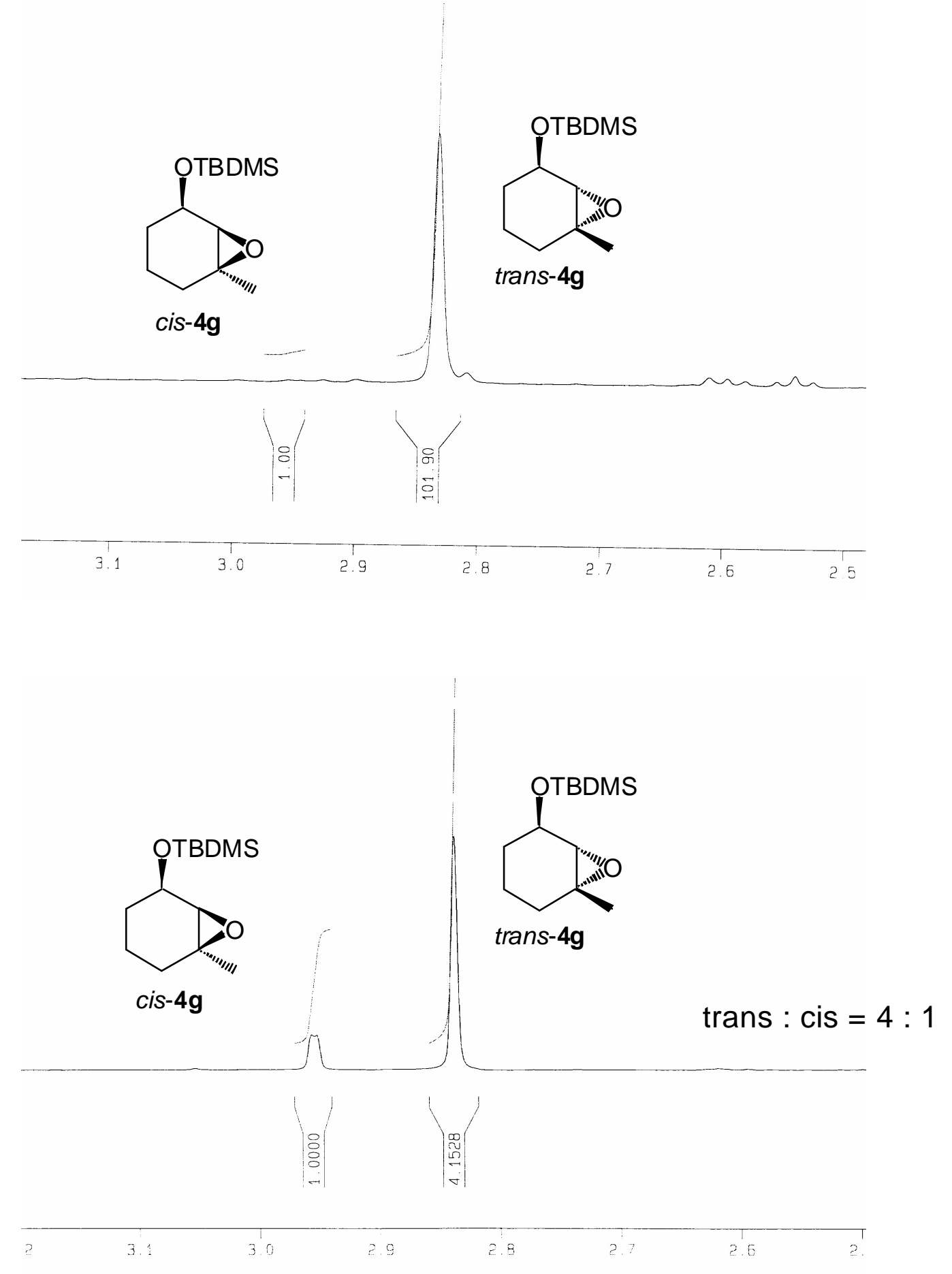
Table 1, entry 8
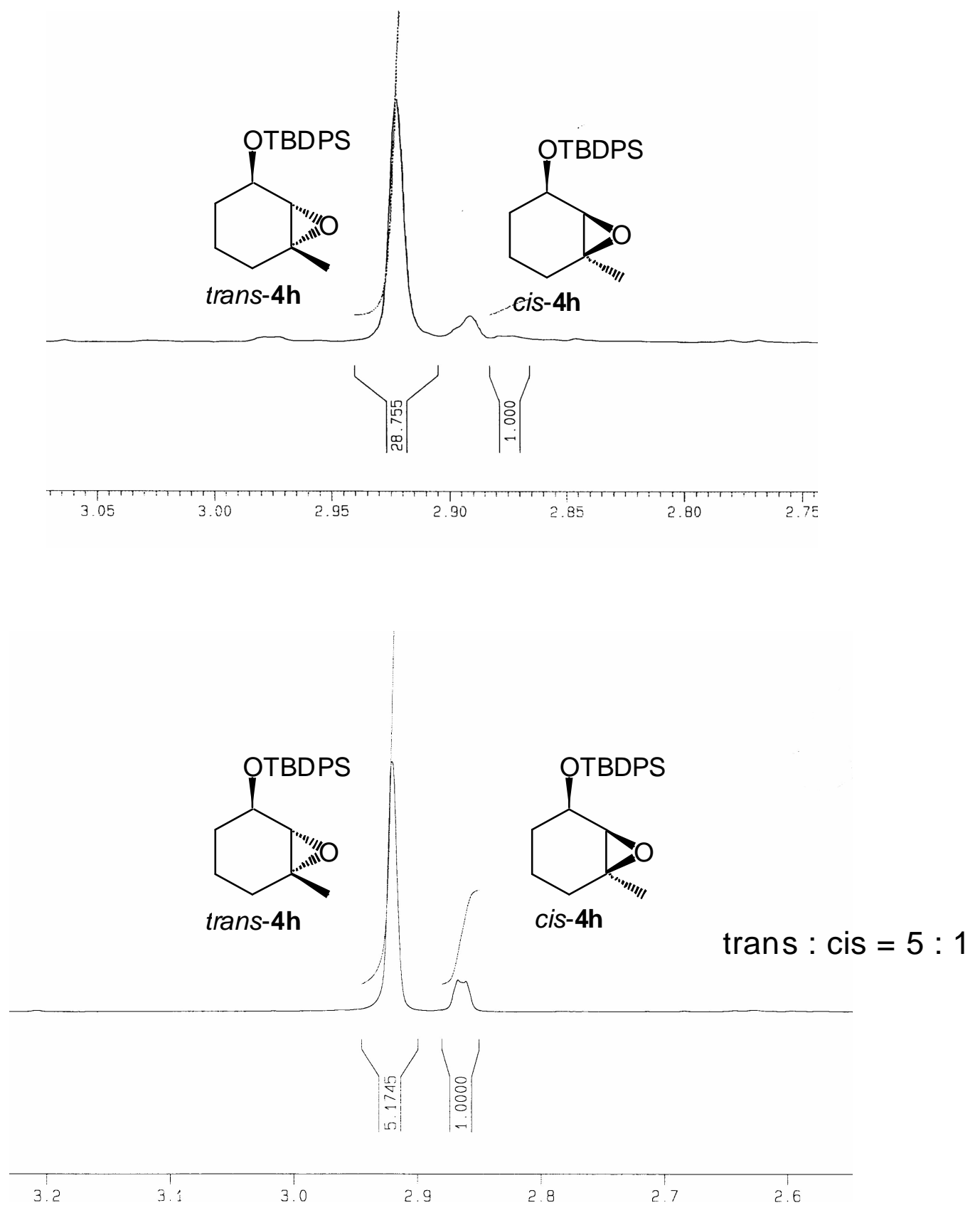
Table 1 , entry 10

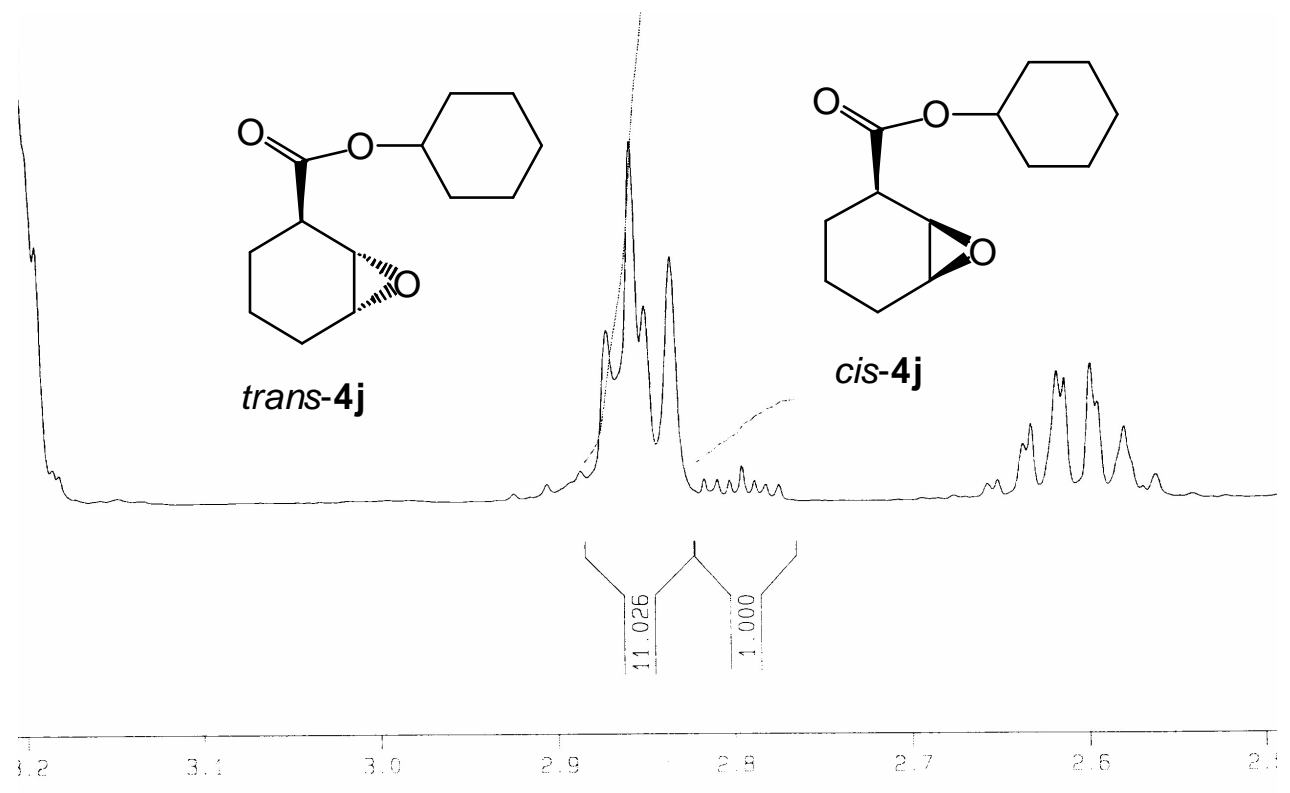

Table 1, entry 11

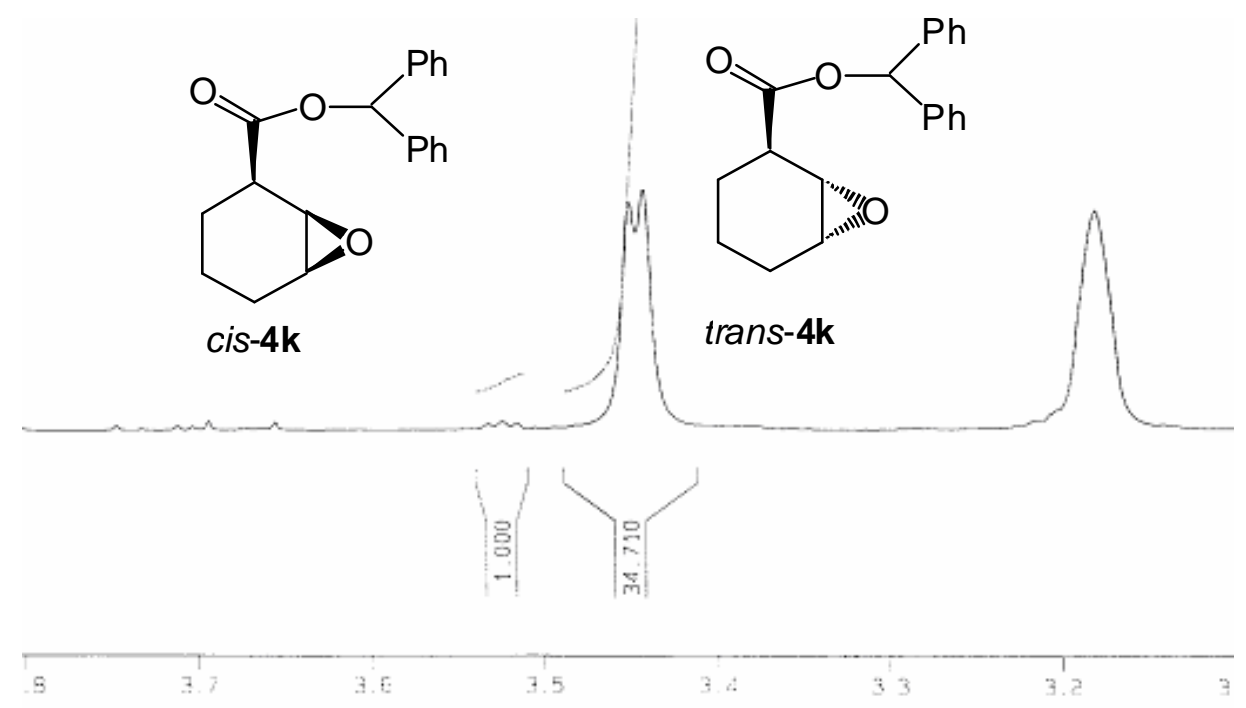


Table 1 , entry 13

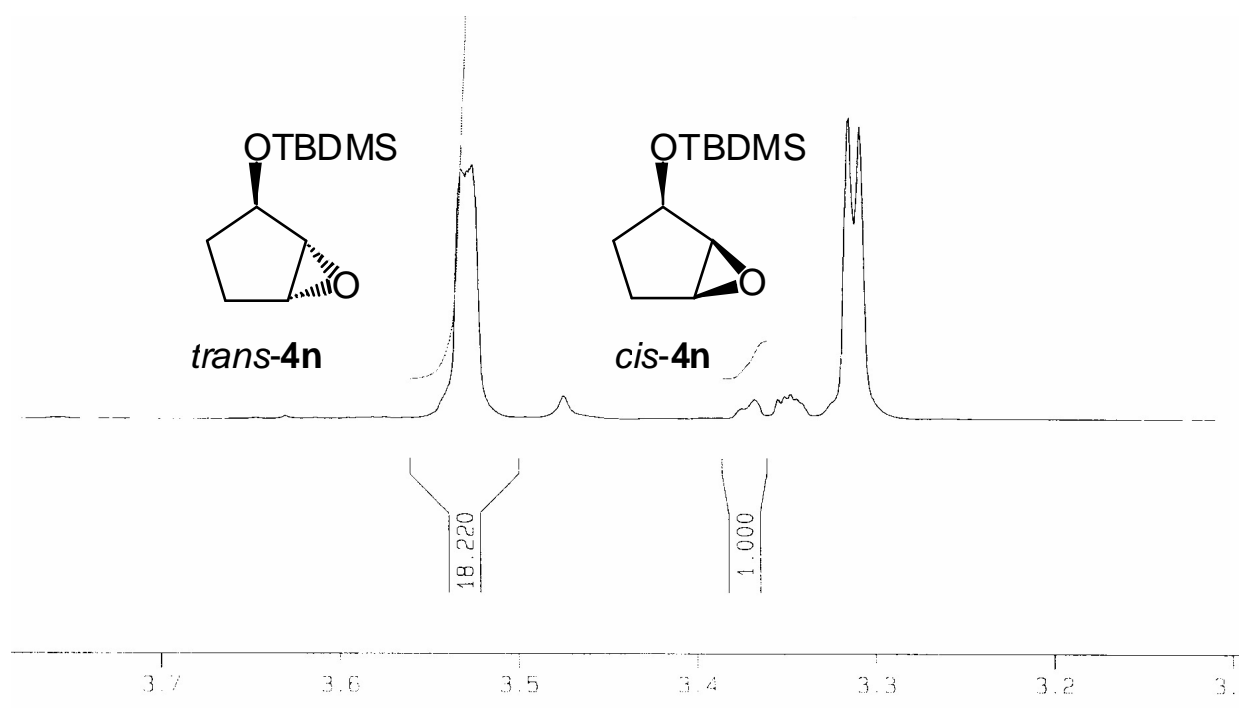

Table 1 , entry 14

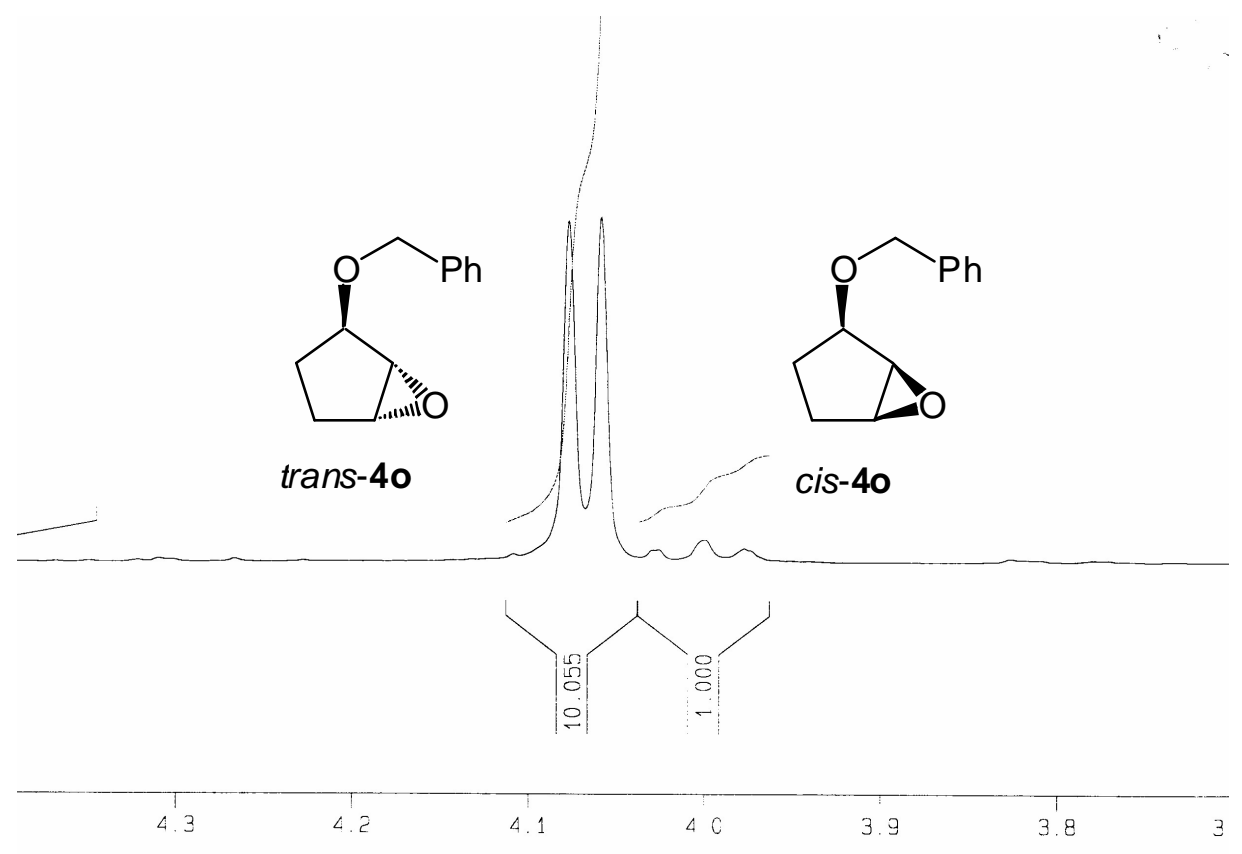


Table 3, entry 2
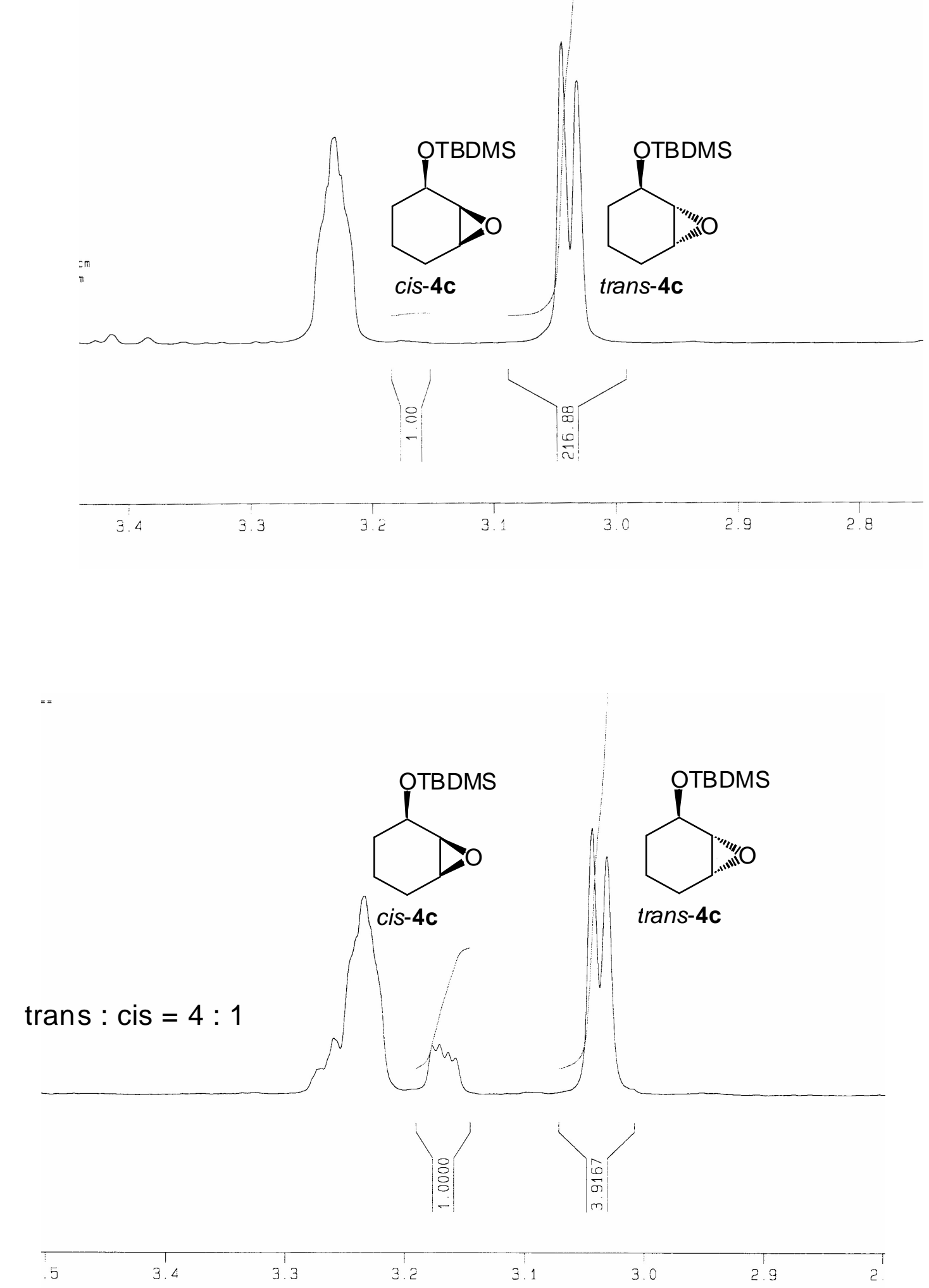
Table 3, entry 3

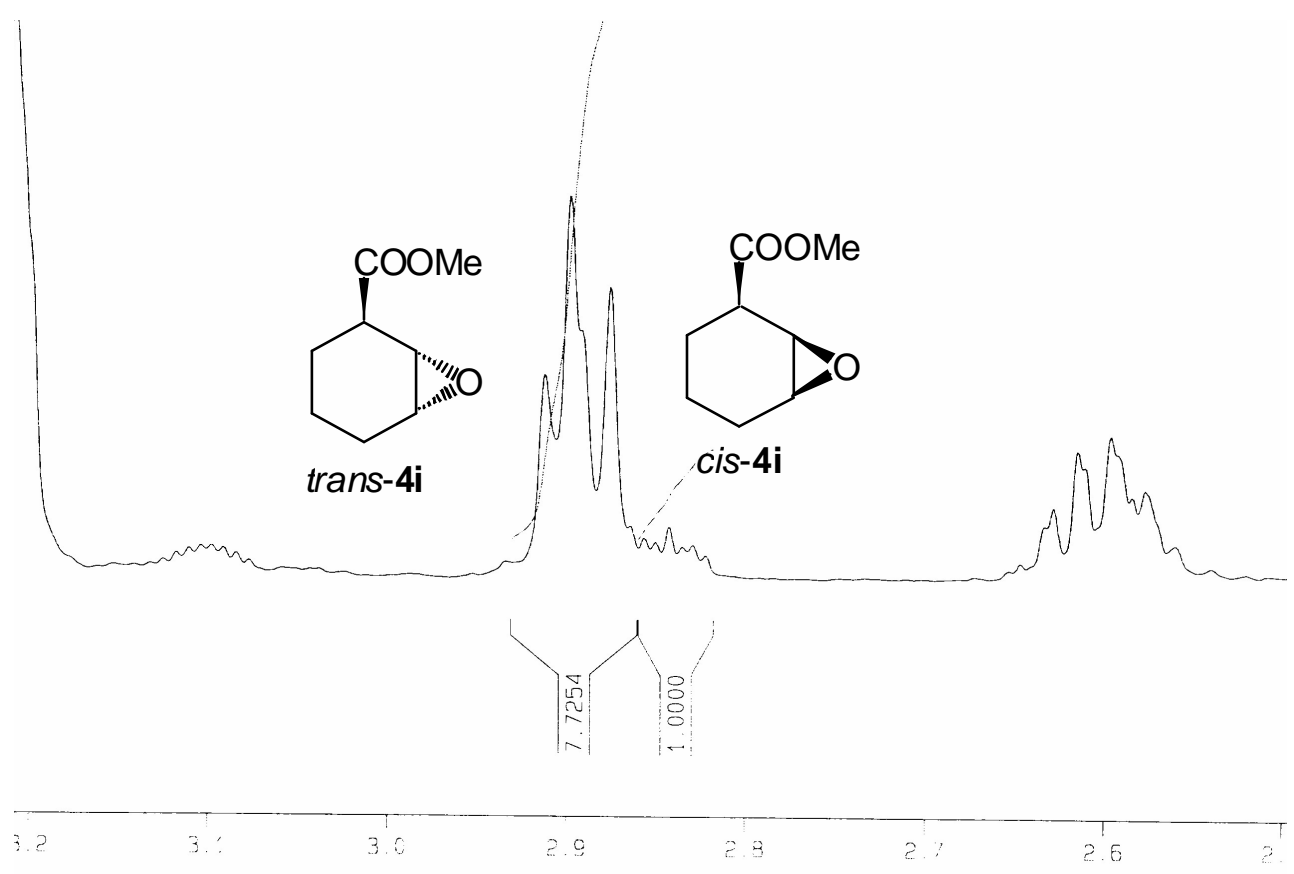


Table 3, entry 4
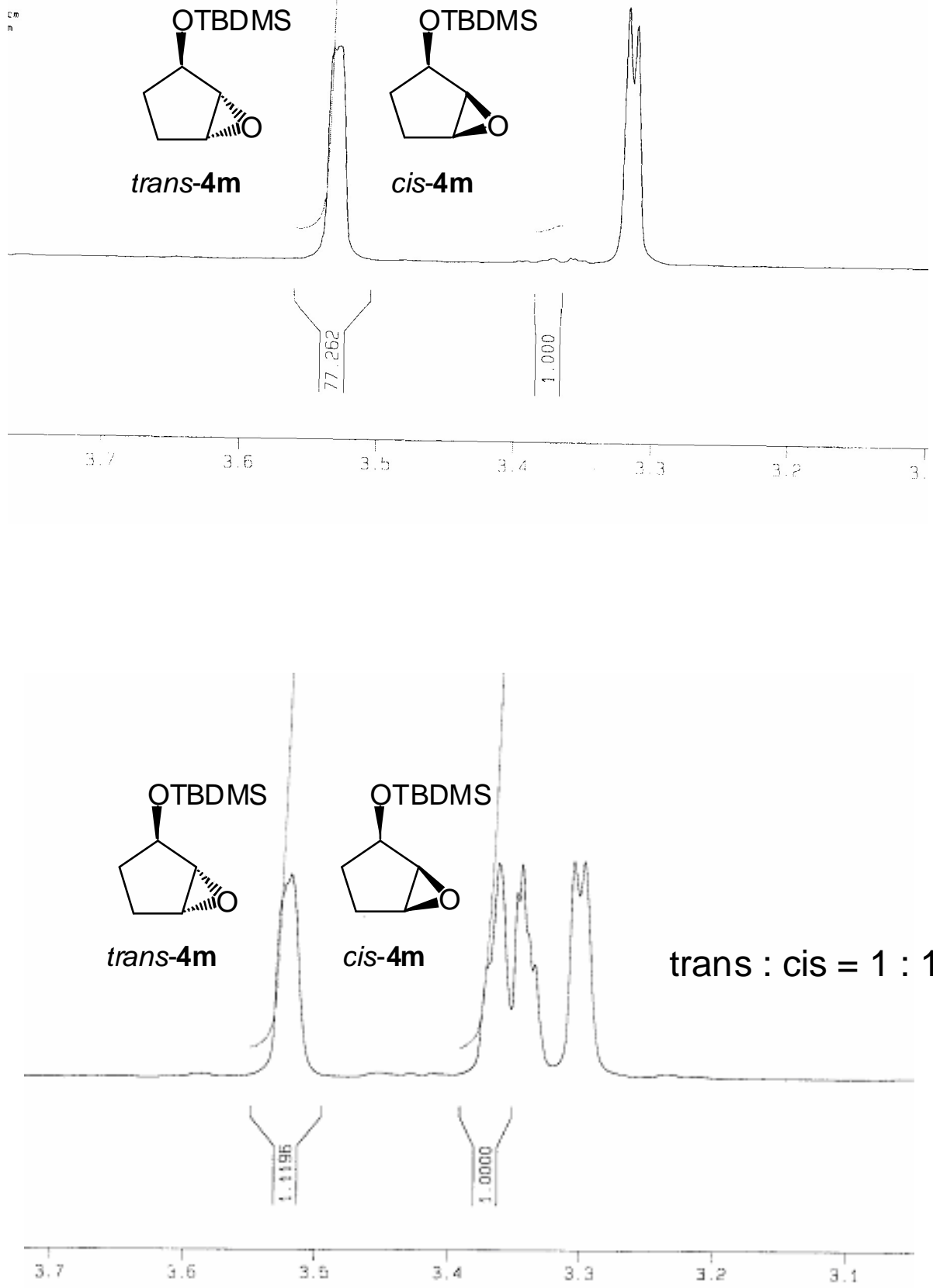
Table 3, entry 6

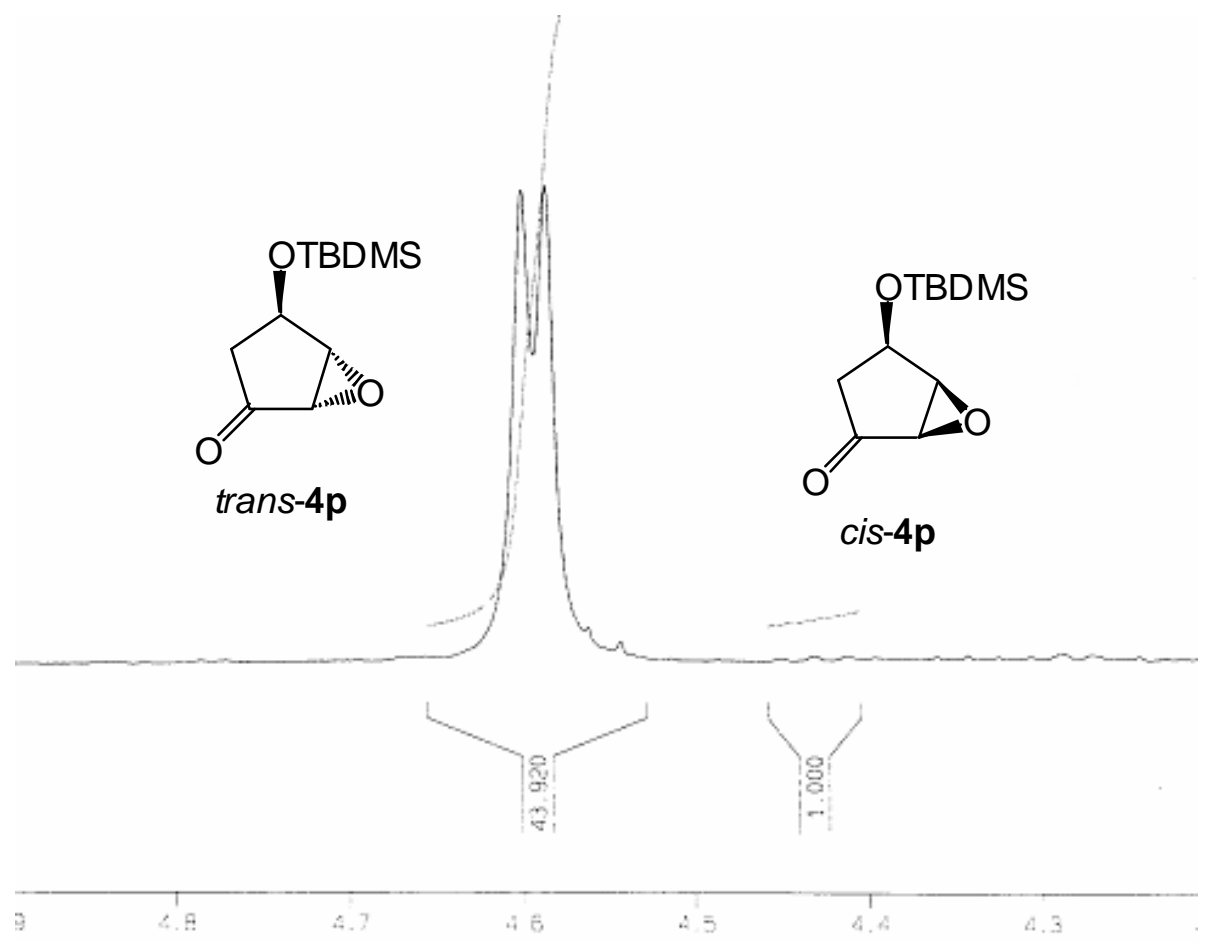




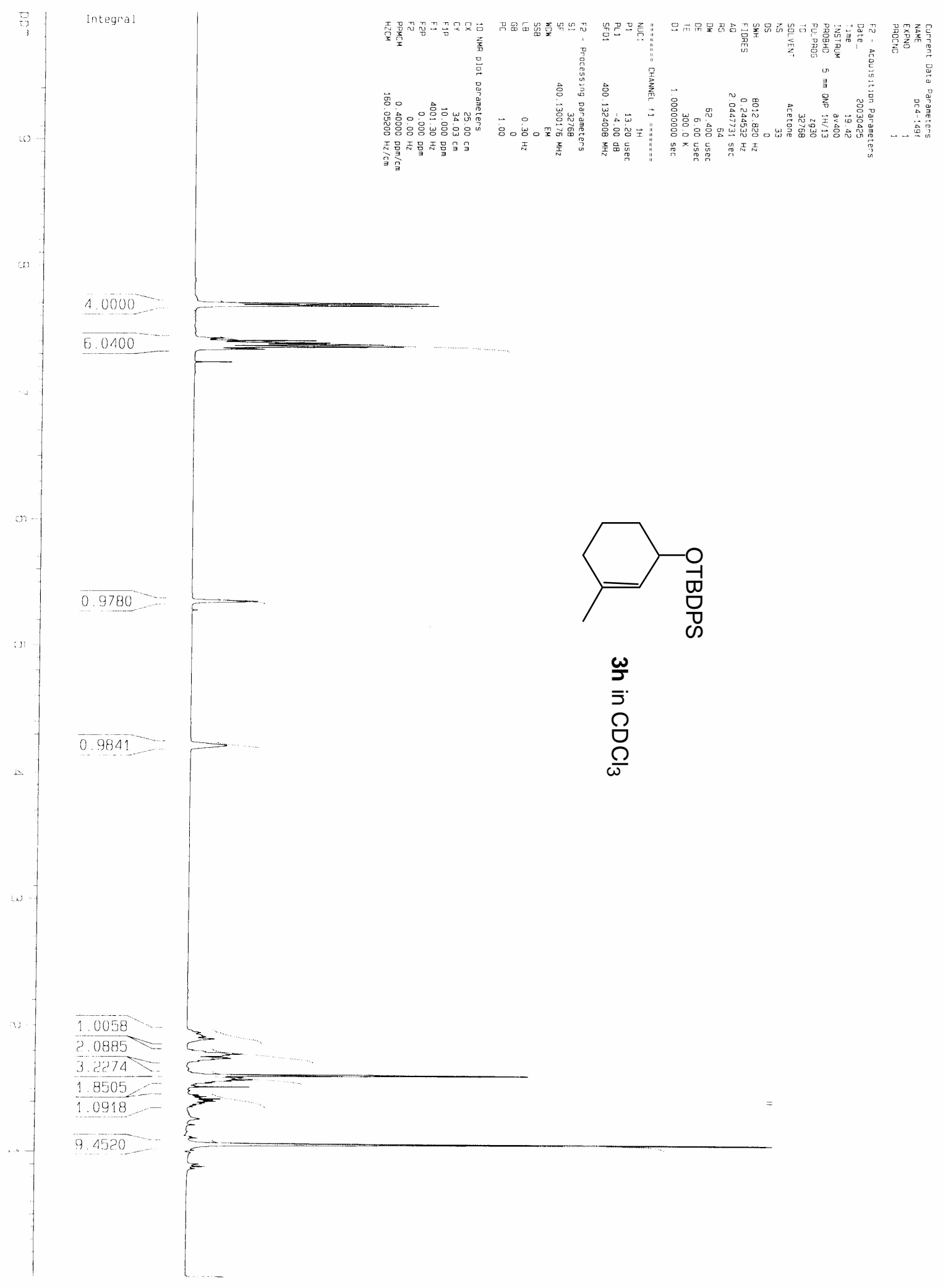




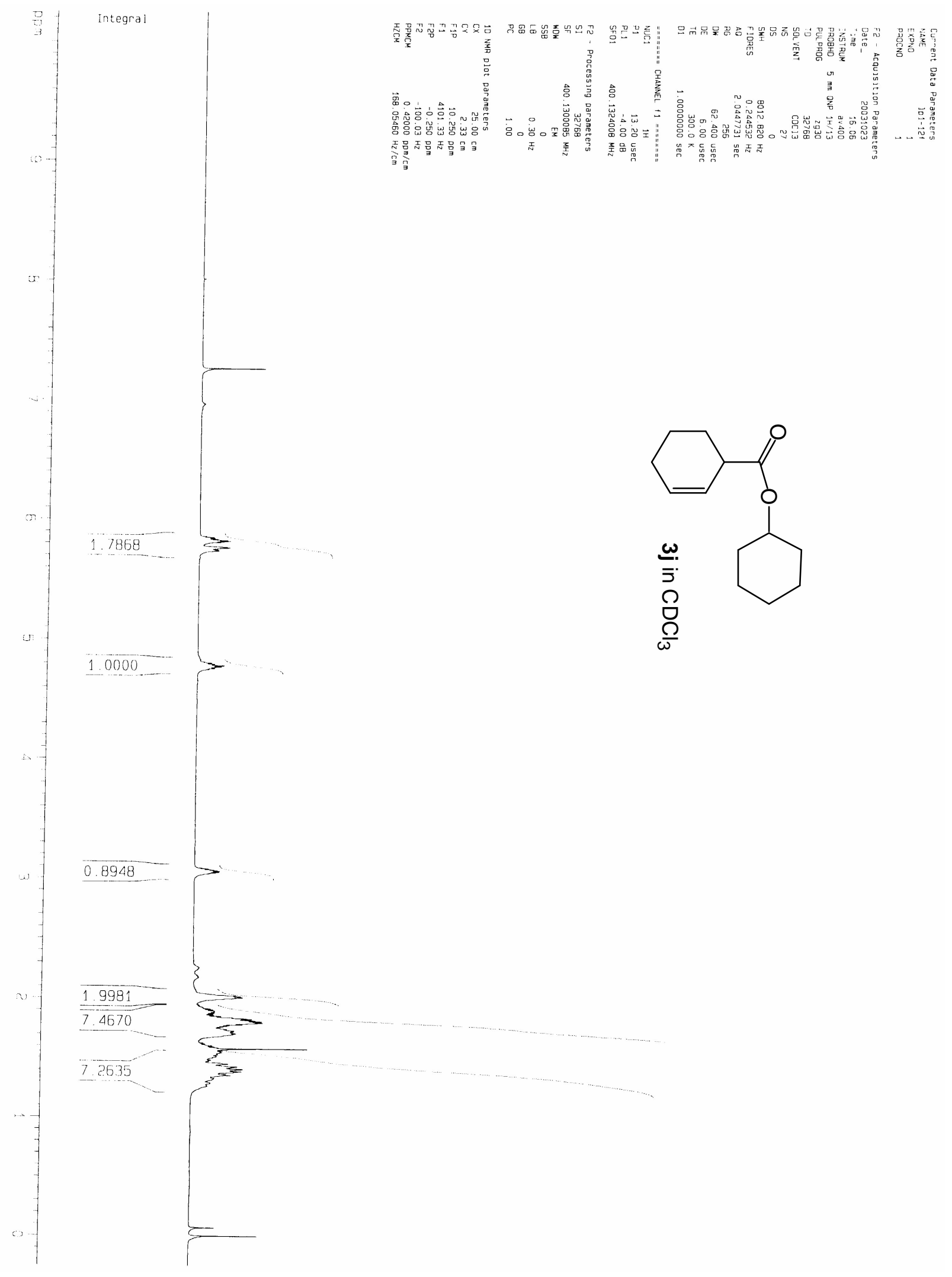




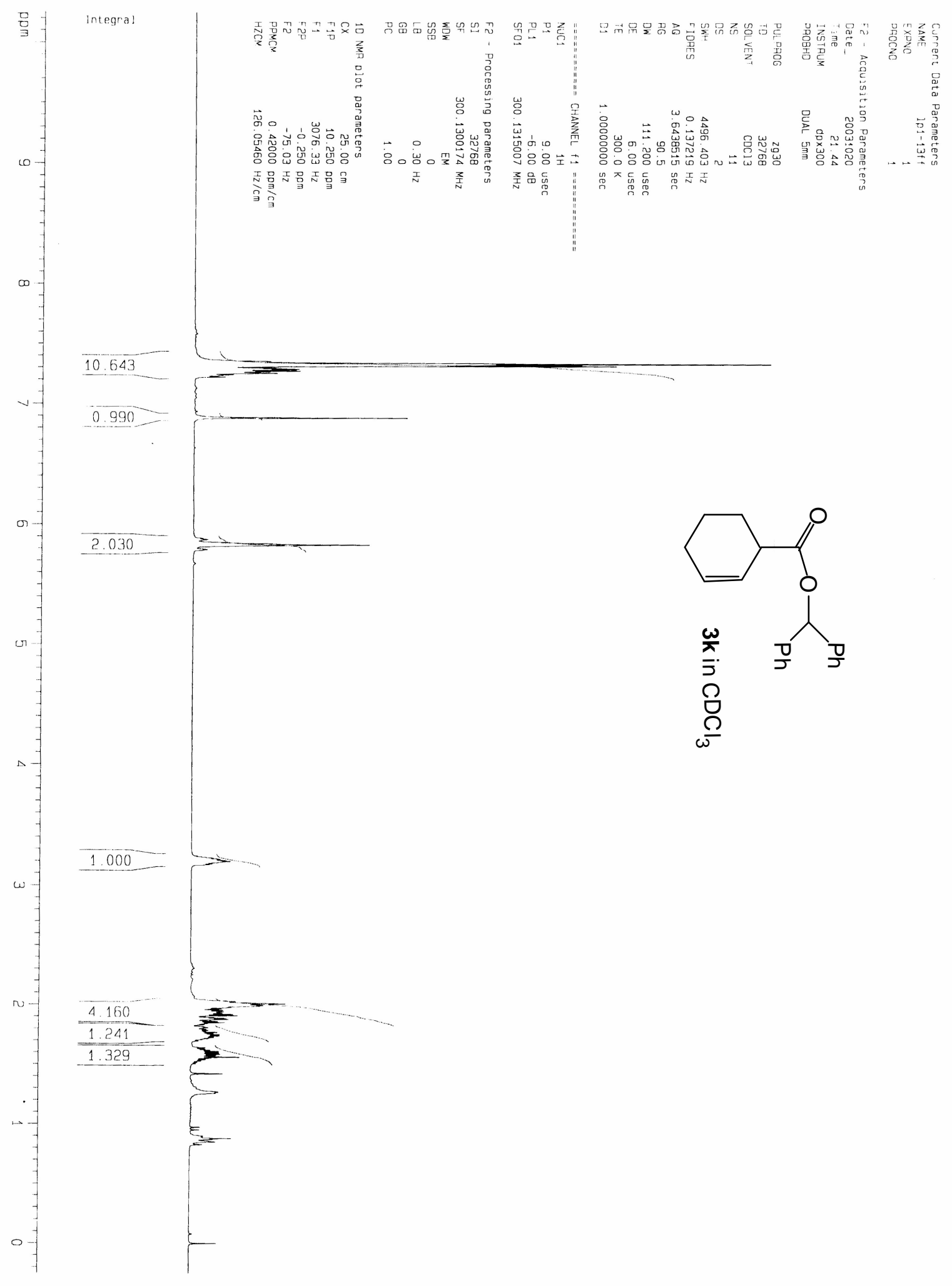




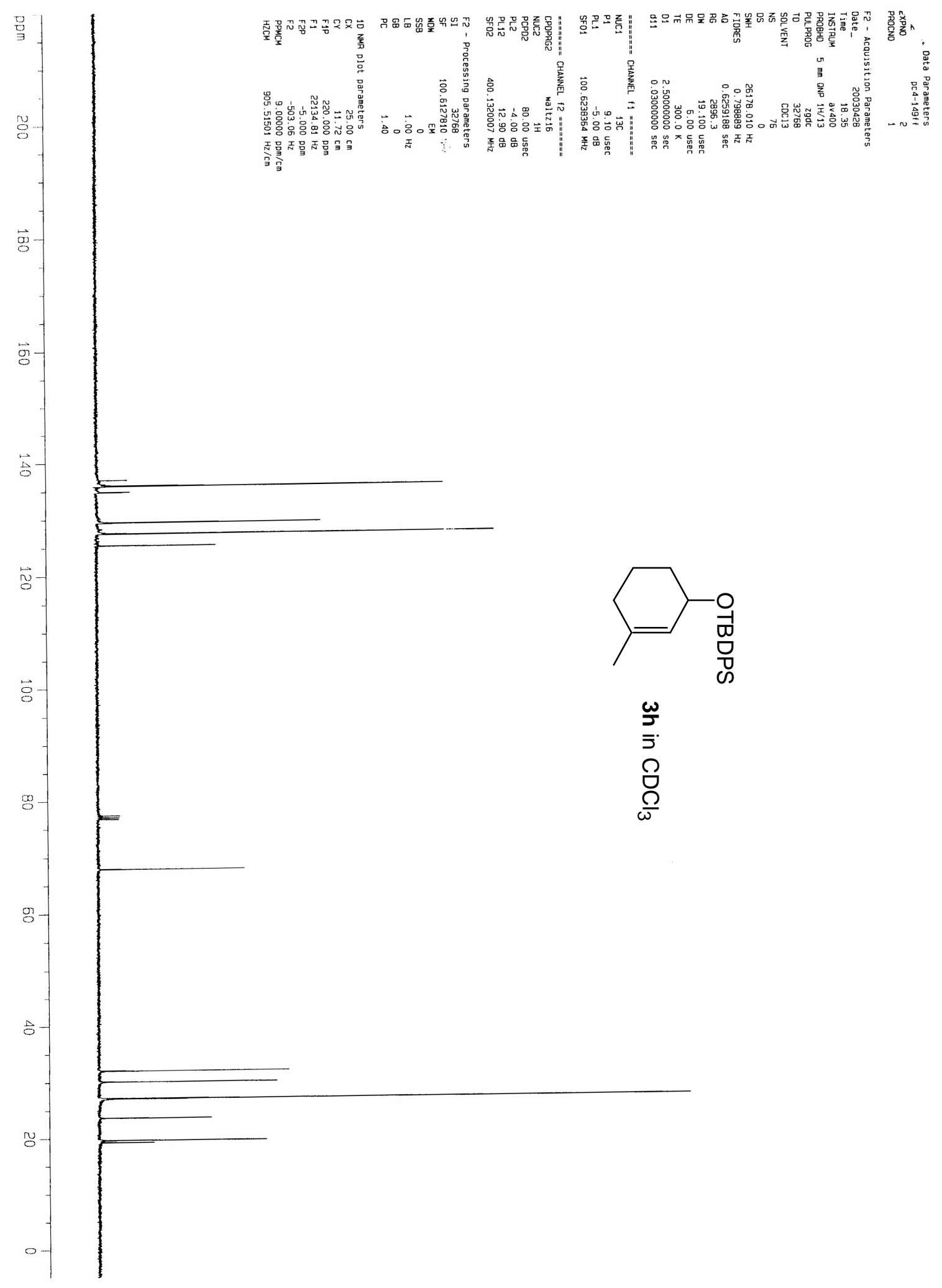




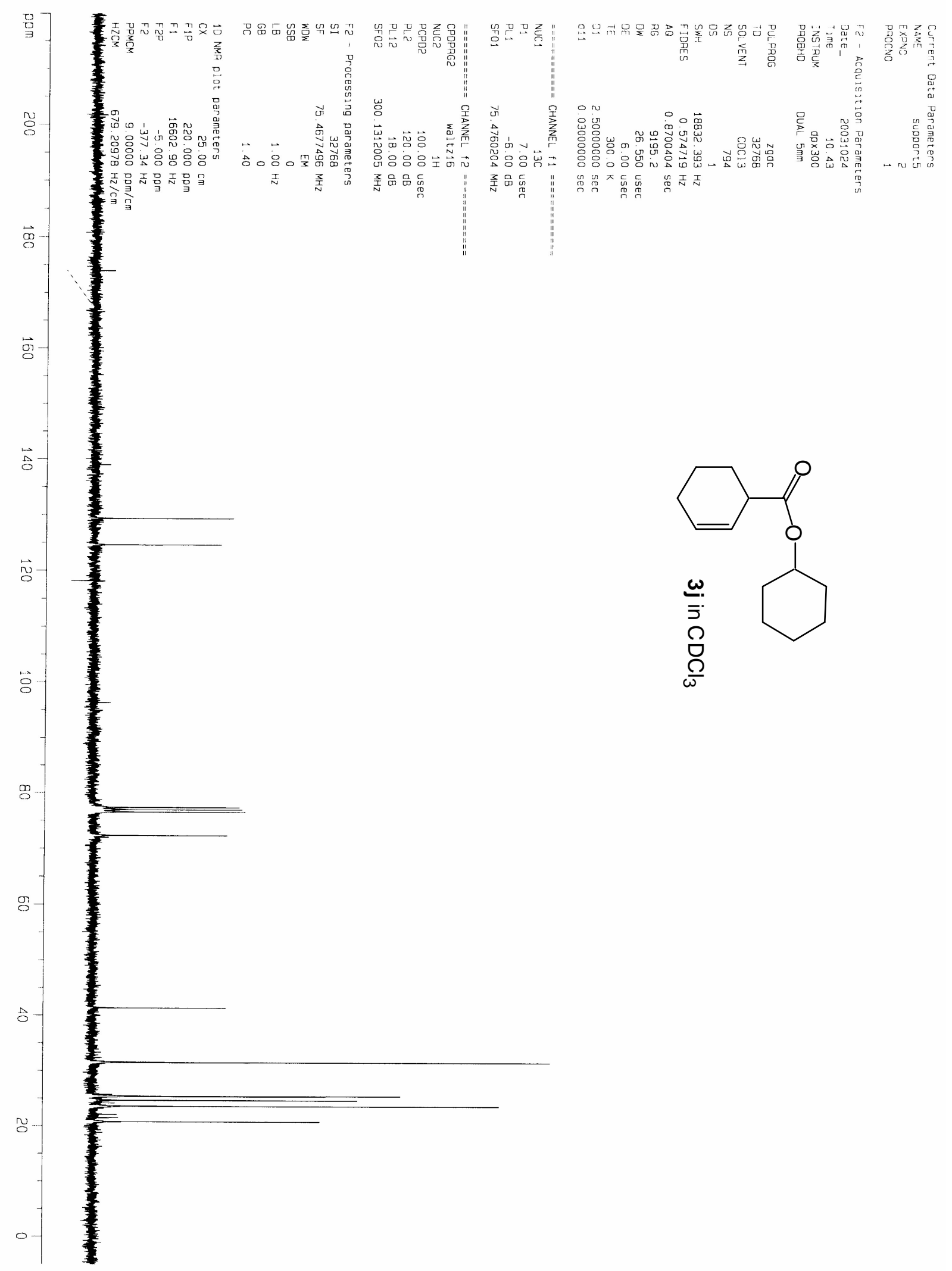




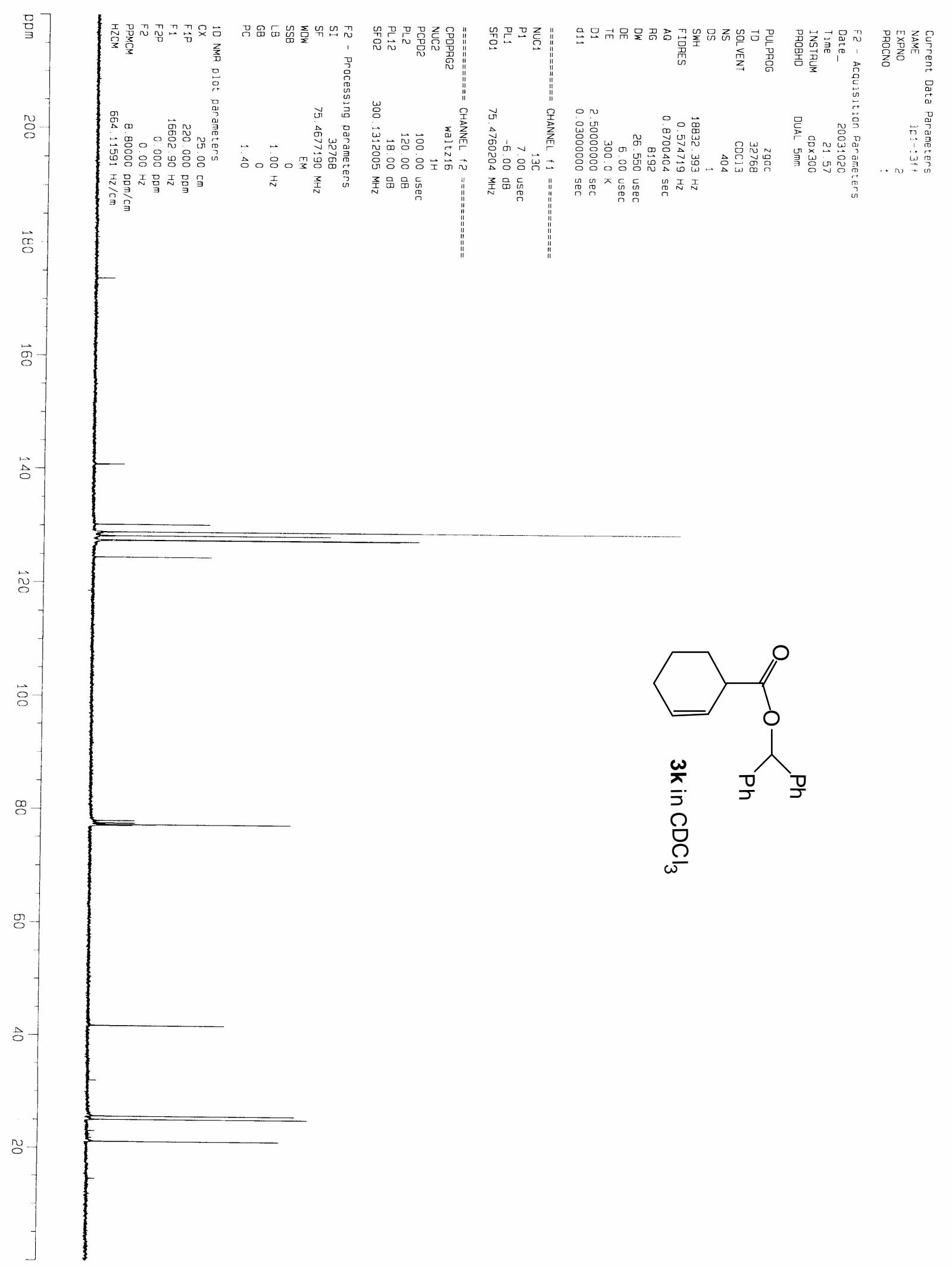




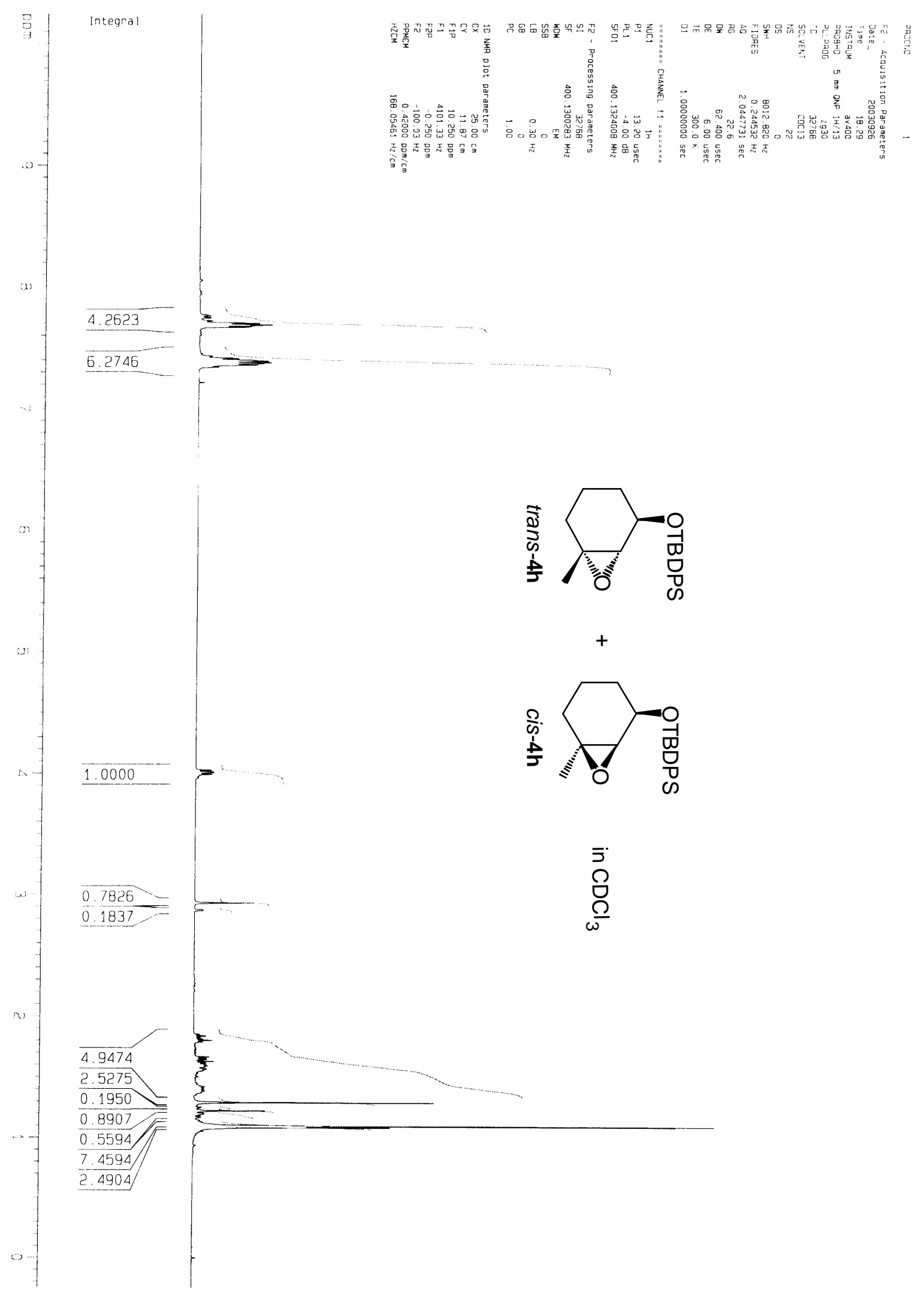




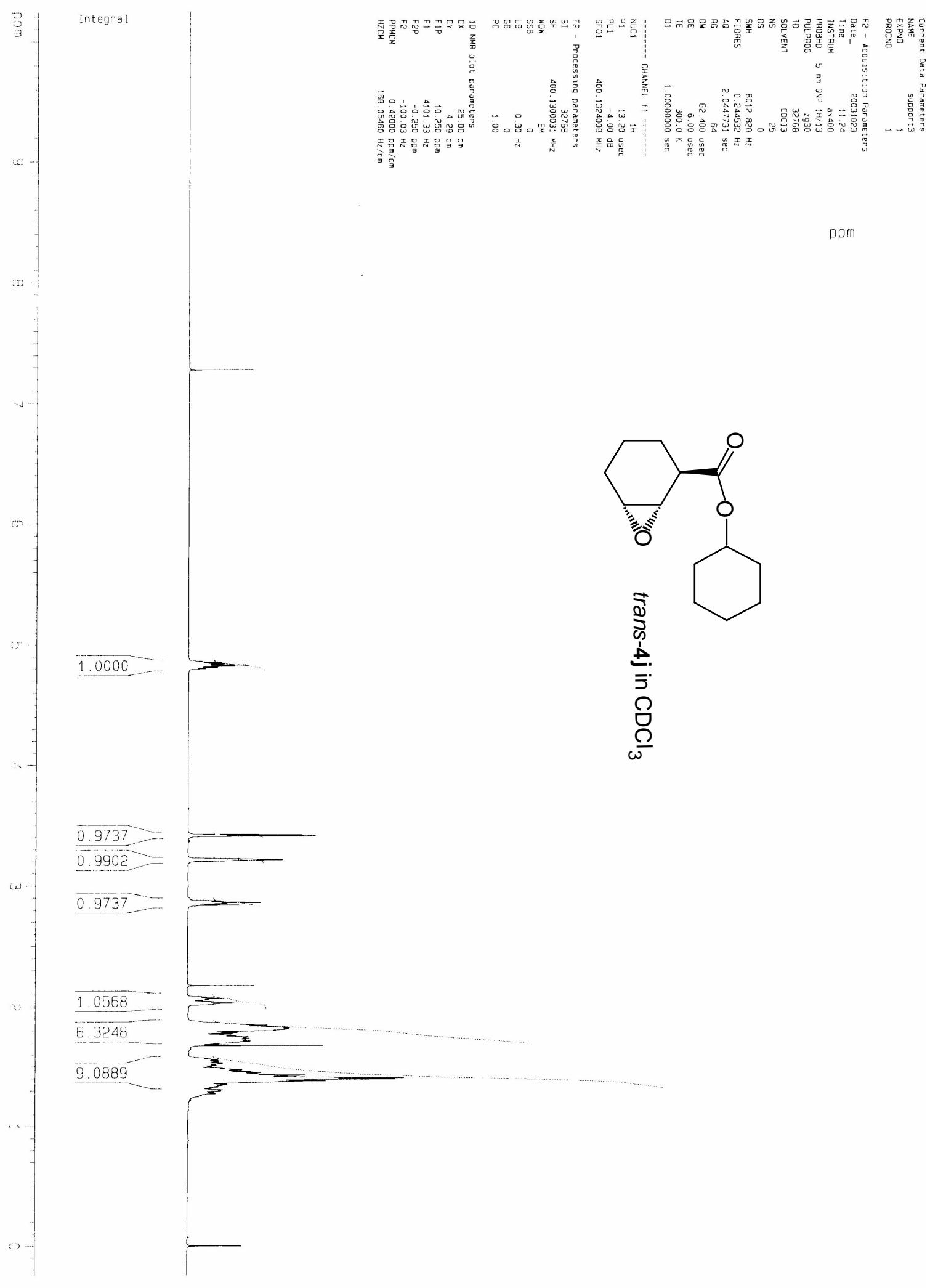



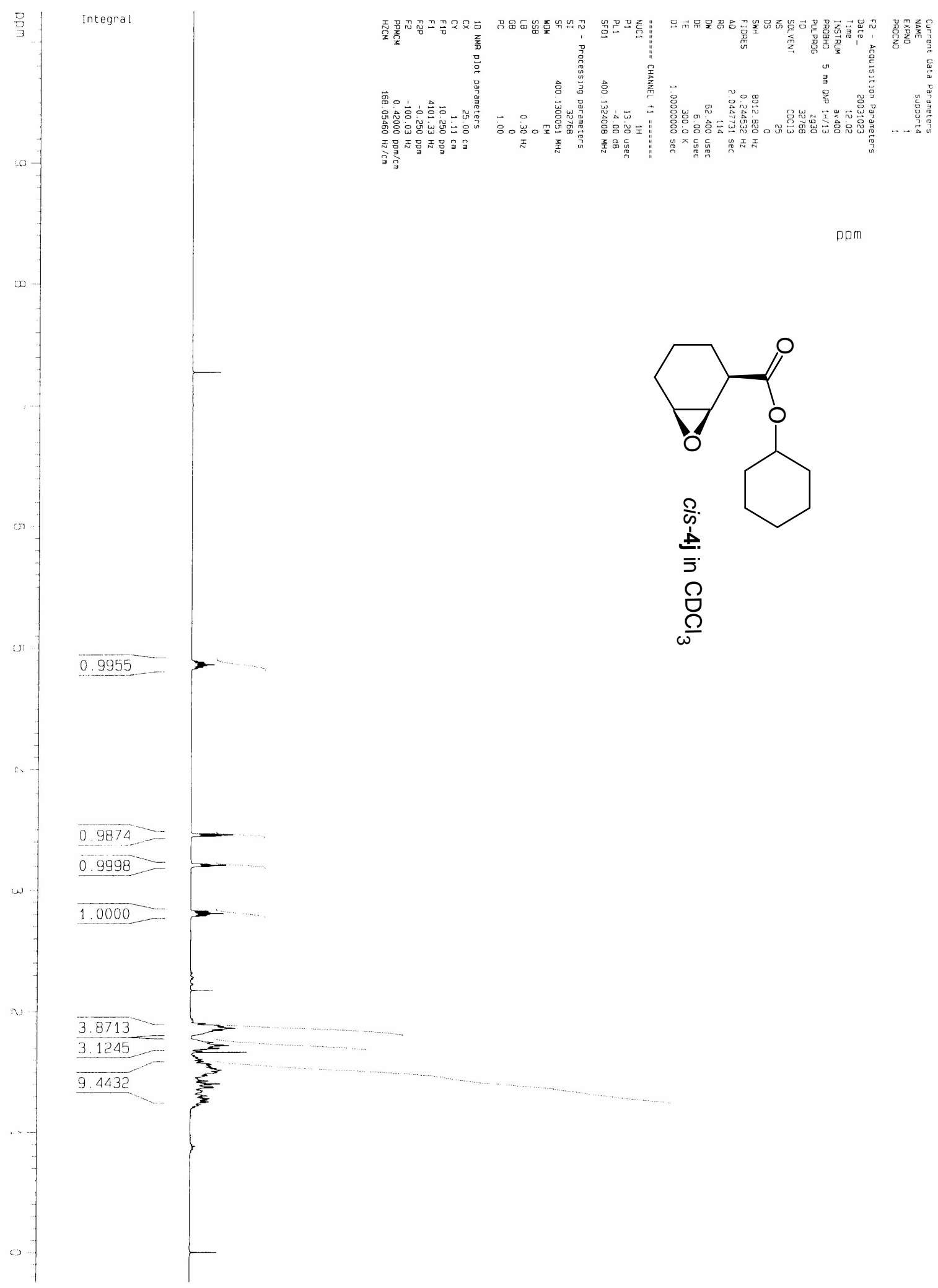

ppm

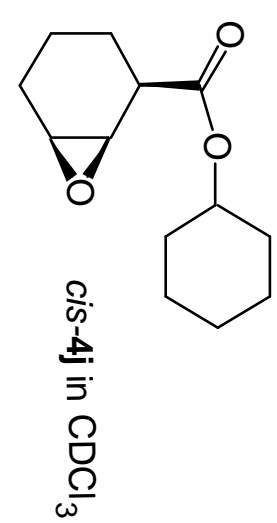




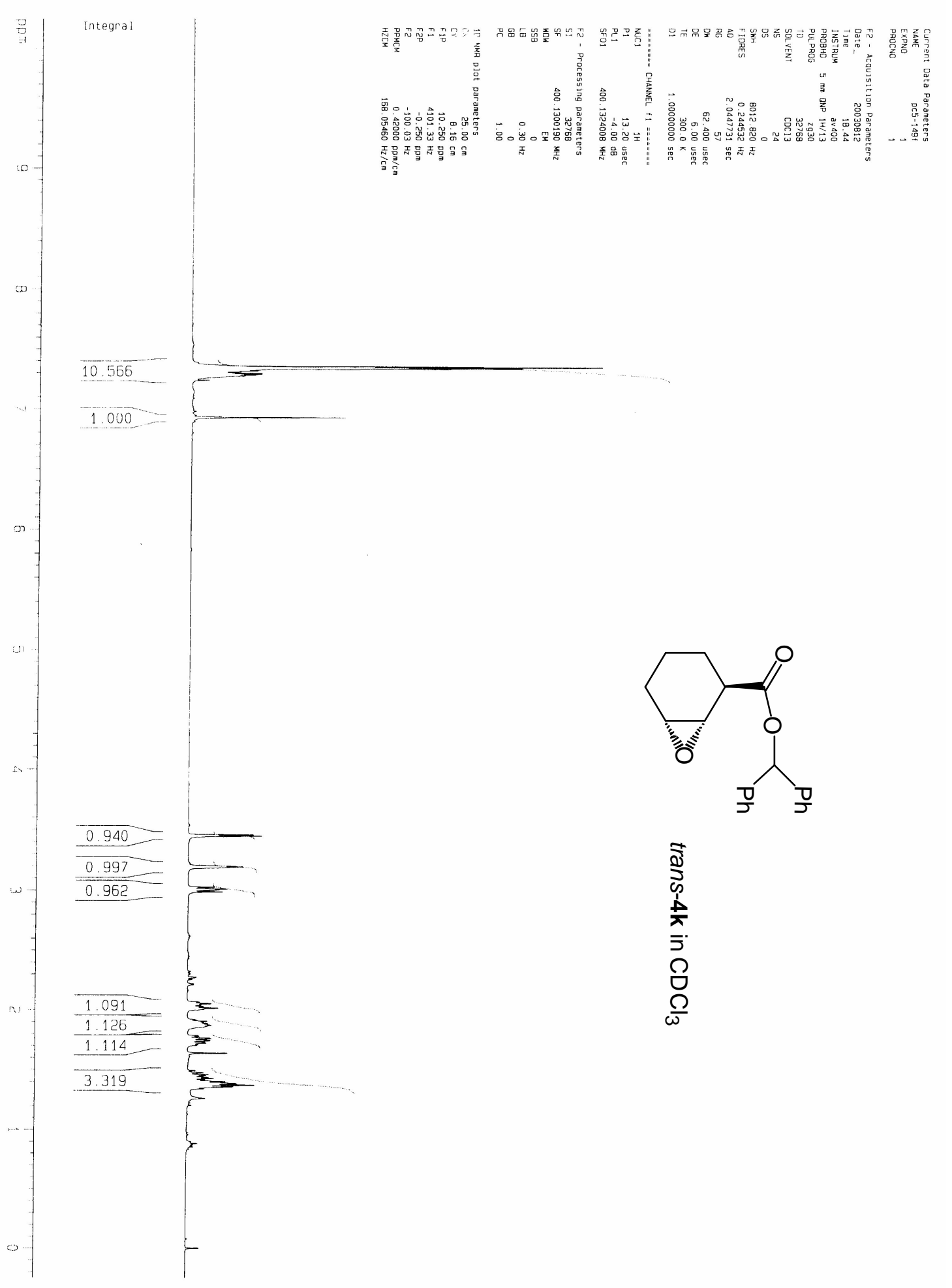




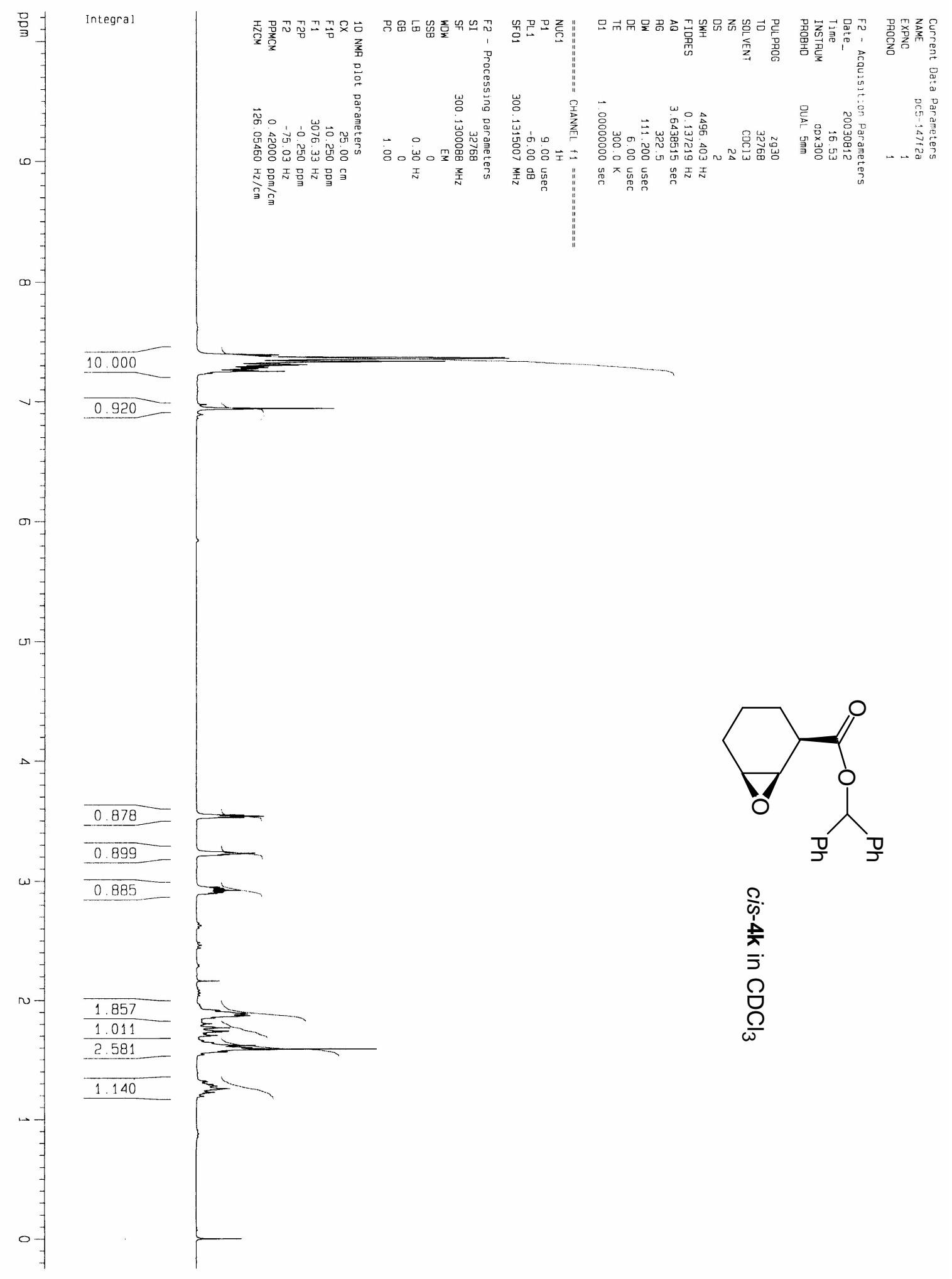




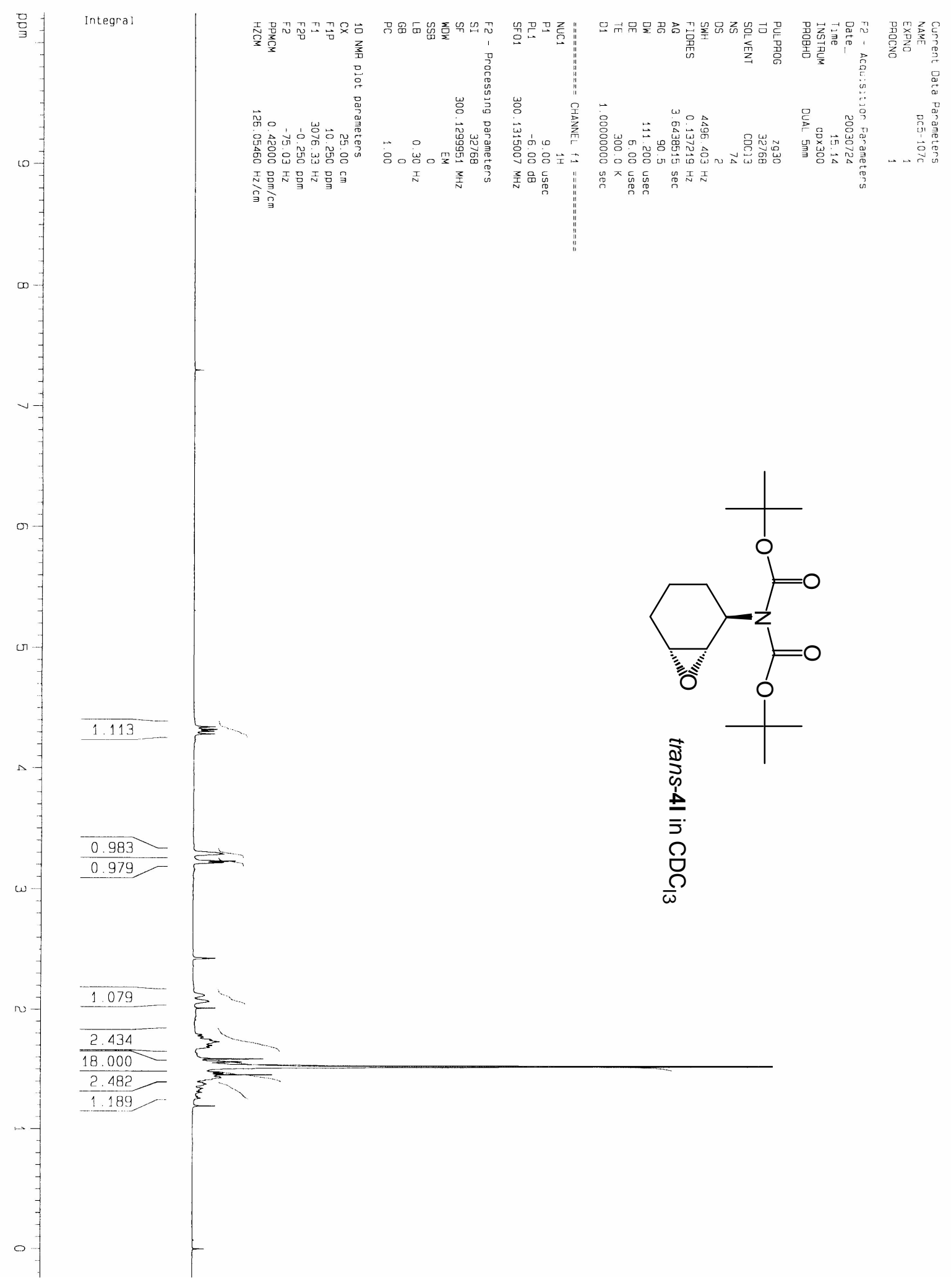




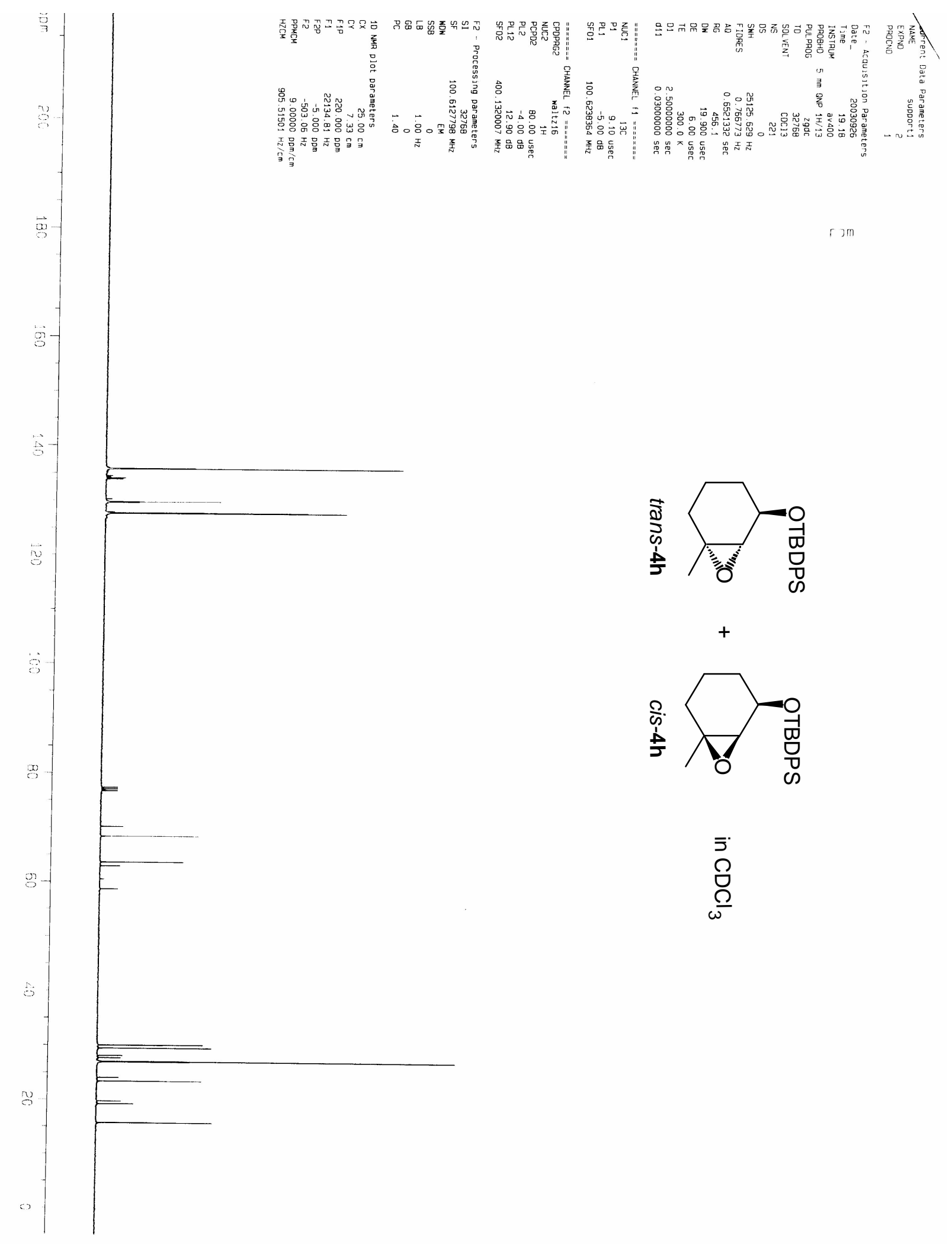




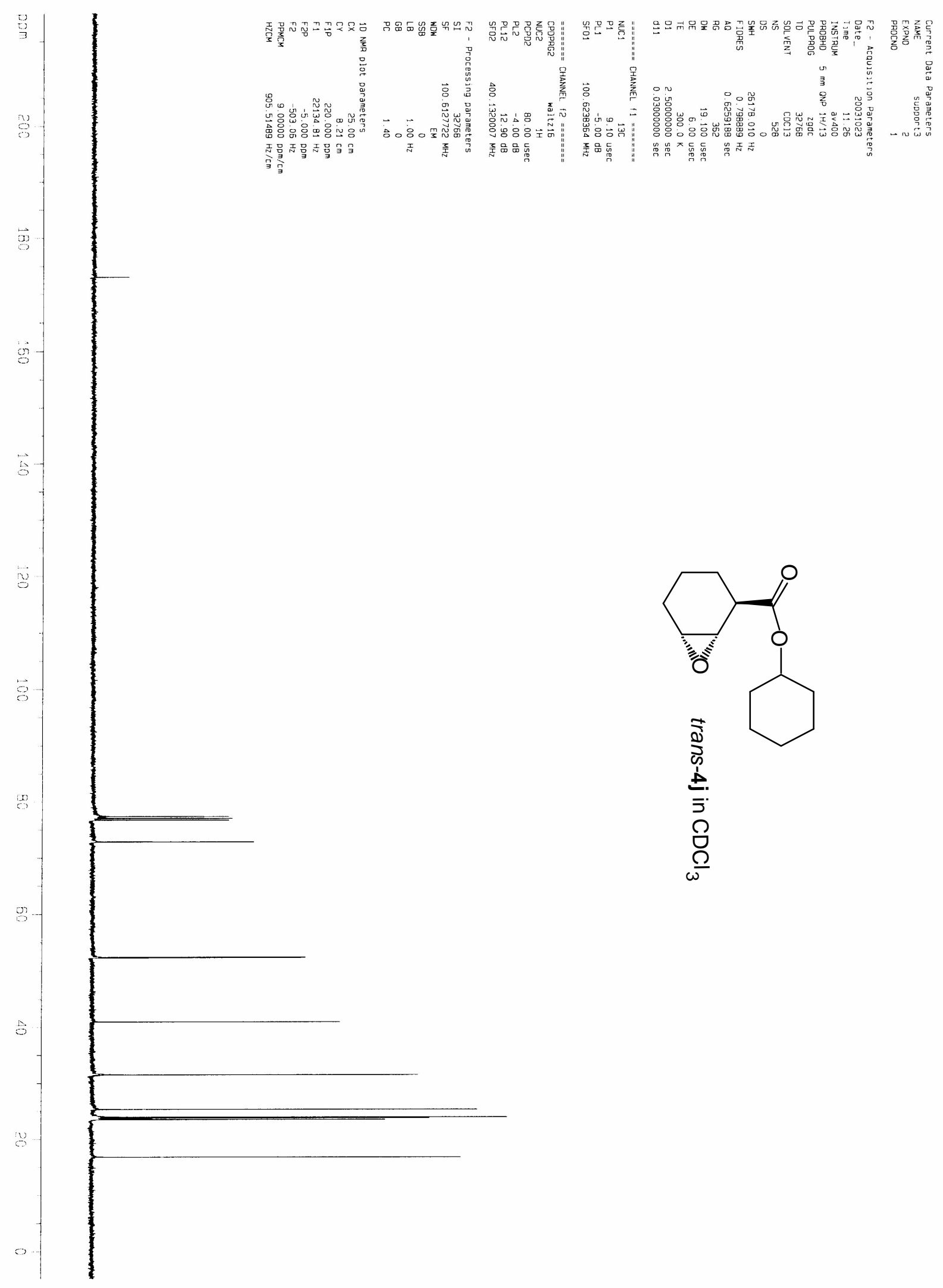




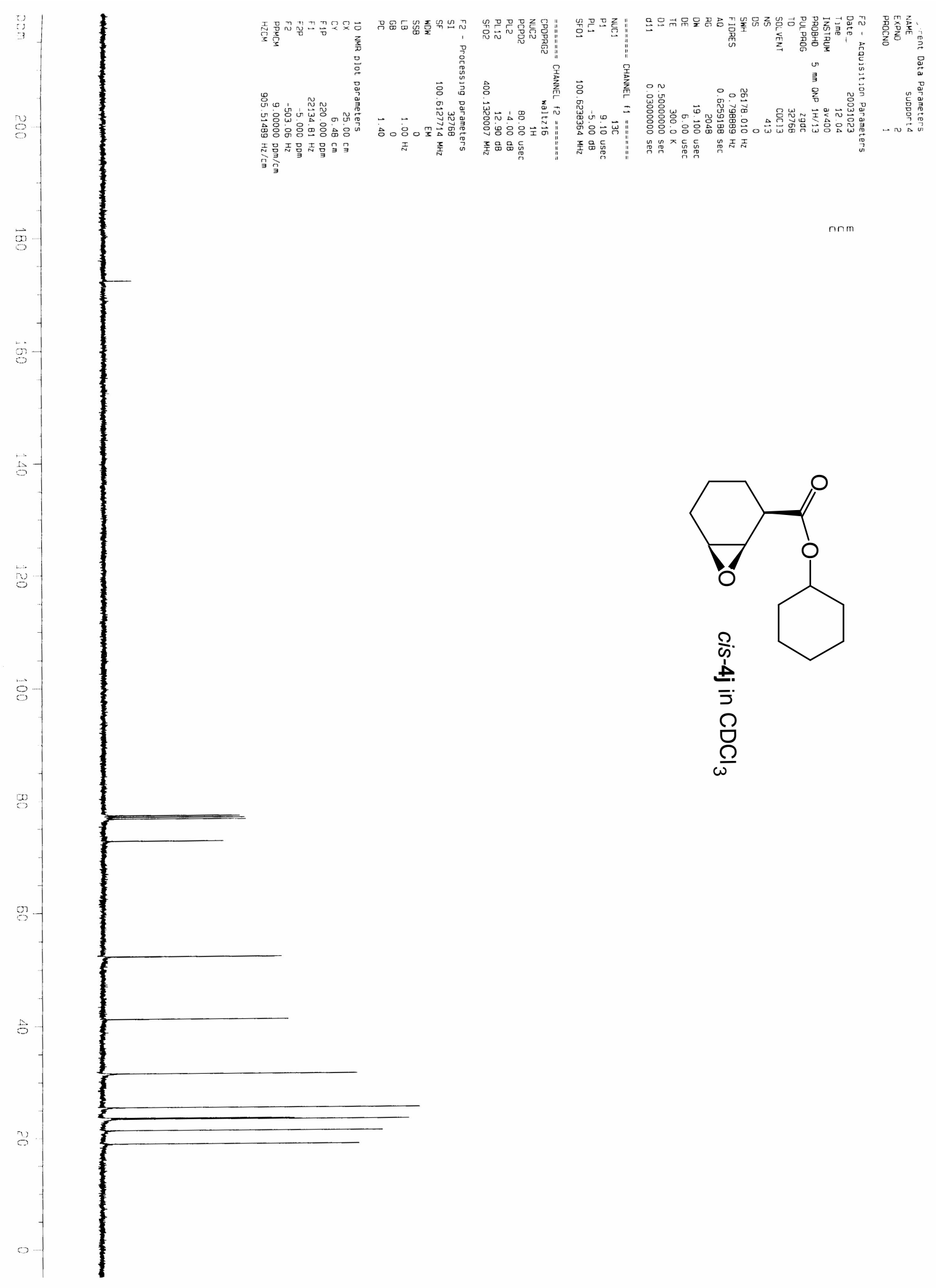




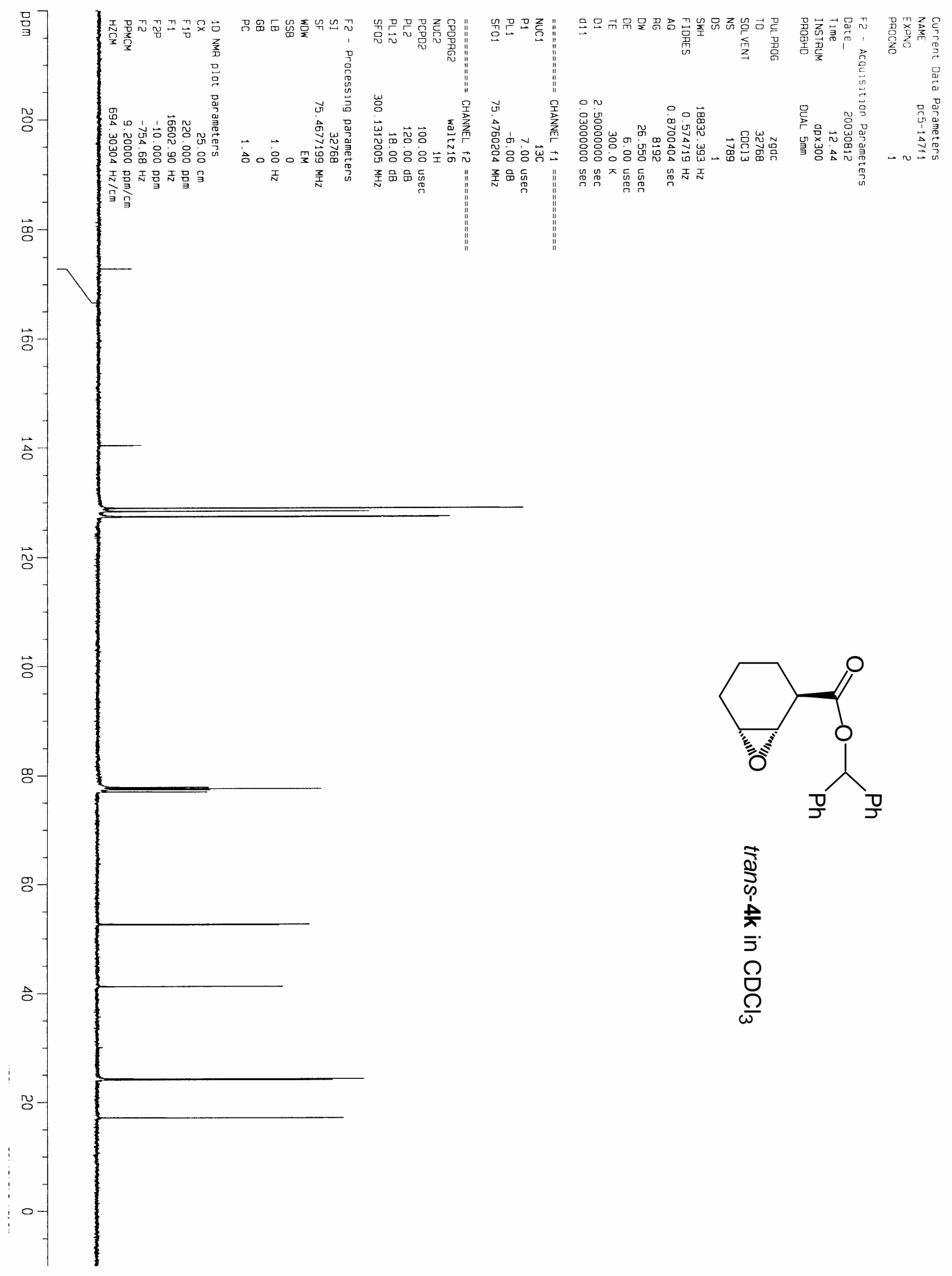




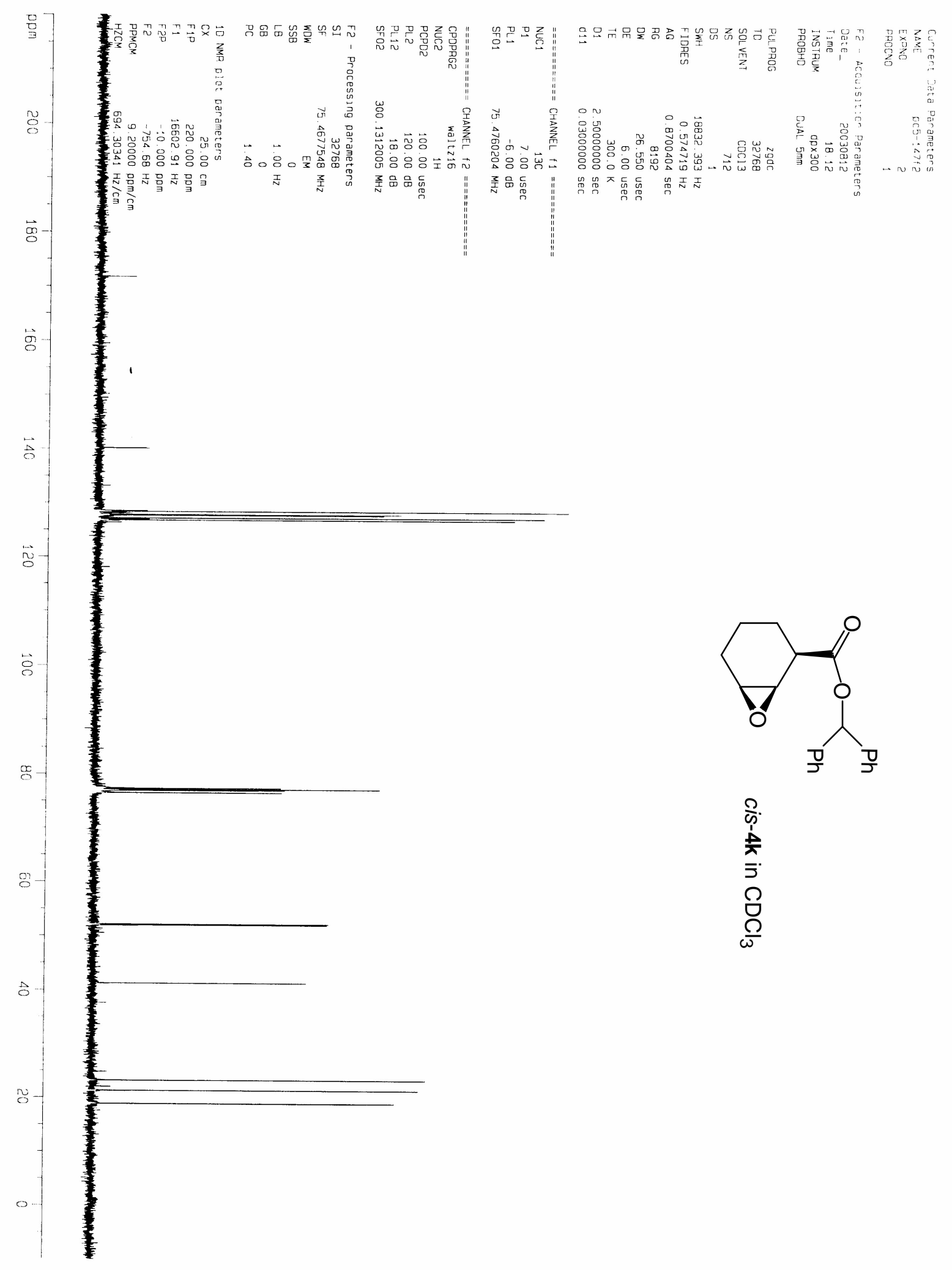




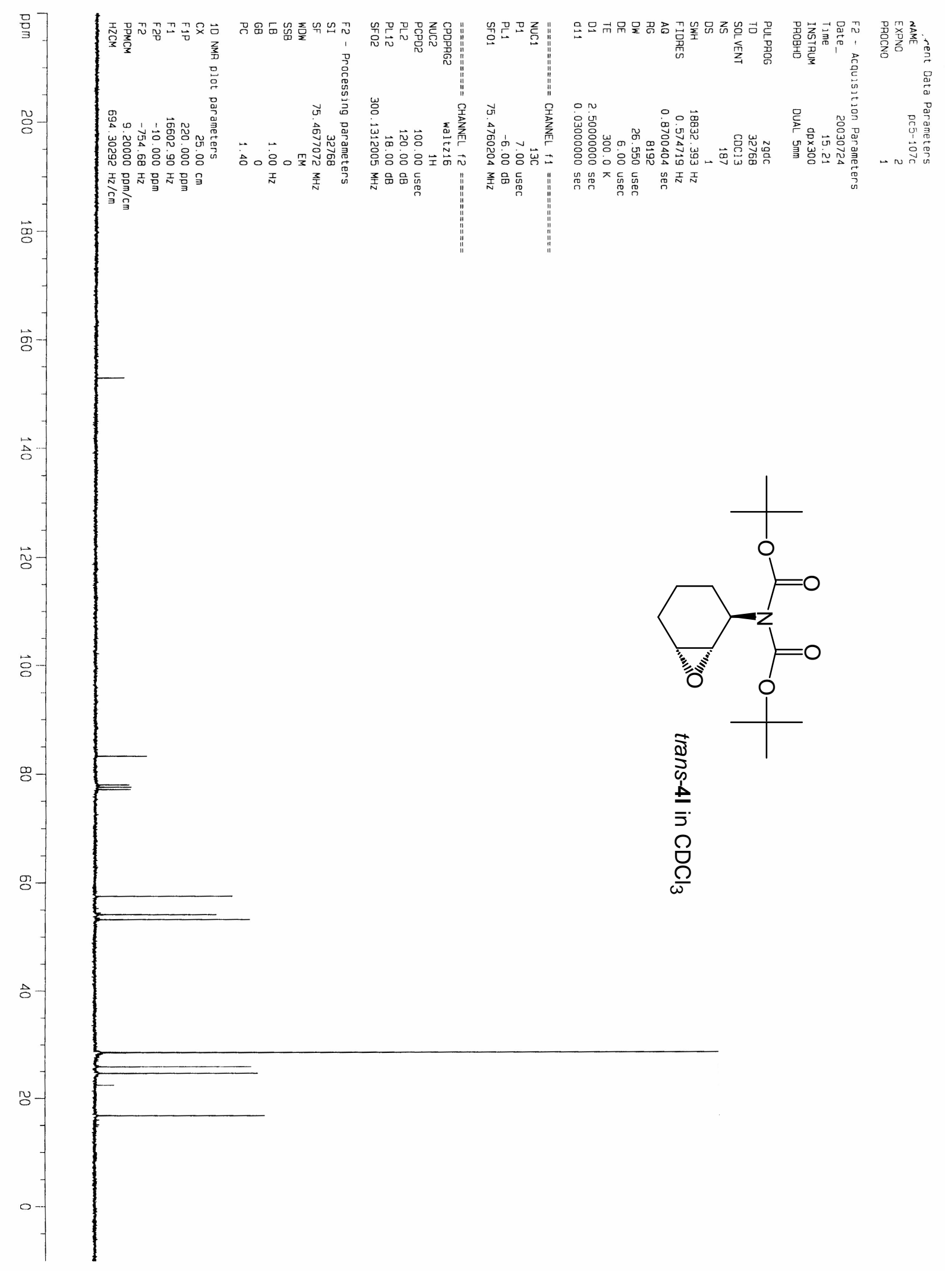

